\title{
BISWAS T.
}

\section{$(p, q)$ TH ORDER ORIENTED GROWTH MEASUREMENT OF COMPOSITE $p$-ADIC ENTIRE FUNCTIONS}

\begin{abstract}
Let $\mathbb{K}$ be a complete ultrametric algebraically closed field and let $\mathcal{A}(\mathbb{K})$ be the $\mathbb{K}$-algebra of entire functions on $\mathbb{K}$. For any $p$-adic entire function $f \in \mathcal{A}(\mathbb{K})$ and $r>0$, we denote by $|f|(r)$ the number sup $\{|f(x)|:|x|=r\}$, where $|\cdot|(r)$ is a multiplicative norm on $\mathcal{A}(\mathbb{K})$. For any two entire functions $f \in \mathcal{A}(\mathbb{K})$ and $g \in \mathcal{A}(\mathbb{K})$ the ratio $\frac{|f|(r)}{|g|(r)}$ as $r \rightarrow \infty$ is called the comparative growth of $f$ with respect to $g$ in terms of their multiplicative norms. Likewise to complex analysis, in this paper we define the concept of $(p, q)$ th order (respectively $(p, q)$ th lower order) of growth as $\rho^{(p, q)}(f)=\limsup _{r \rightarrow+\infty} \frac{\log [p]|f|(r)}{\log ^{[q]} r}$ (respectively $\lambda^{(p, q)}(f)=\liminf _{r \rightarrow+\infty} \frac{\log ^{[p]}|f|(r)}{\log ^{\mid q]} r}$ ), where $p$ and $q$ are any two positive integers. We study some growth properties of composite $p$-adic entire functions on the basis of their $(p, q)$ th order and $(p, q)$ th lower order.

Key words and phrases: $p$-adic entire function, growth, $(p, q)$ th order, $(p, q)$ th lower order, composition.
\end{abstract}

Rajbari, Rabindrapalli, R. N. Tagore Road, P.O. Krishnagar, Dist-Nadia, 741101, West Bengal, India

E-mail: tanmaybiswas_math@rediffmail.com

\section{INTRODUCTION AND DEFINITIONS}

Let $\mathbb{K}$ be an algebraically closed field of characteristic 0 , complete with respect to a $p$-adic absolute value $|\cdot|$ (example $\mathbb{C}_{p}$ ). For any $\alpha \in \mathbb{K}$ and $R \in(0,+\infty)$, the closed disk $\{x \in \mathbb{K}$ : $|x-\alpha| \leq R\}$ and the open disk $\{x \in \mathbb{K}:|x-\alpha|<R\}$ are denoted by $d(\alpha, R)$ and $d\left(\alpha, R^{-}\right)$ respectively. Also $C(\alpha, r)$ denotes the circle $\{x \in \mathbb{K}:|x-\alpha|=r\}$. Moreover $\mathcal{A}(\mathbb{K})$ represent the $\mathbb{K}$-algebra of analytic functions on $\mathbb{K}$, i.e. the set of power series with an infinite radius of convergence. For the most comprehensive study of analytic functions inside a disk or in the whole field $\mathbb{K}$, we refer the reader to the books $[9,10,15,18]$. During the last several years the ideas of $p$-adic analysis have been studied from different aspects and many important results were gained (see [1-6], [8,11-14,19]).

Let $f \in \mathcal{A}(\mathbb{K})$ and $r>0$, then we denote by $|f|(r)$ the number $\sup \{|f(x)|:|x|=r\}$ where $|\cdot|(r)$ is a multiplicative norm on $\mathcal{A}(\mathbb{K})$. For any two entire functions $f \in \mathcal{A}(\mathbb{K})$ and $g \in \mathcal{A}(\mathbb{K})$ the ratio $\frac{|f|(r)}{|g|(r)}$ as $r \rightarrow \infty$ is called the growth of $f$ with respect to $g$ in terms of their multiplicative norms.

For any $x \in[0, \infty)$ and $k \in \mathbb{N}$, we define recursively $\log ^{[k]} x=\log \left(\log ^{[k-1]} x\right)$ and $\exp ^{[k]} x=\exp \left(\exp ^{[k-1]} x\right)$, where $\mathbb{N}$ stands for the set of all positive integers. We also denote $\log ^{[0]} x=x$ and $\exp ^{[0]} x=x$. Throughout the paper, log denotes the Neperian logarithm. 
Taking this into account the order (resp. lower order) of an entire function $f \in \mathcal{A}(\mathbb{K})$ is given by (see [4])

$$
\begin{aligned}
& \rho(f) \\
& \lambda(f)
\end{aligned}=\lim _{r \rightarrow+\infty} \sup \frac{\log ^{[2]}|f|(r)}{\log r} .
$$

The above definition of order (resp. lower order) does not seem to be feasible if an entire function $f \in \mathcal{A}(\mathbb{K})$ is of order zero. To overcome this situation and in order to study the growth of an entire function $f \in \mathcal{A}(\mathbb{K})$ precisely, one may introduce the concept of logarithmic order (resp. logarithmic lower order) by increasing $\log ^{+}$once in the denominator following the classical definition of logarithmic order (see, for example, [7]). Therefore the logarithmic order $\rho_{\log }(f)$ and logarithmic lower order $\lambda_{\log }(f)$ of an entire function $f \in \mathcal{A}(\mathbb{K})$ are define as

$$
\begin{aligned}
& \rho_{\log }(f) \\
& \lambda_{\log }(f)
\end{aligned}=\lim _{r \rightarrow+\infty} \sup \frac{\log ^{[2]}|f|(r)}{\log ^{[2]} r} .
$$

Further the concept of $(p, q)$ th order ( $p$ and $q$ are any two positive integers with $p \geq q$ ) is not new and was first introduced by Juneja et al. [16,17]. In the line of Juneja et al. [16,17], now we shall introduce the definitions of $(p, q)$ th order and $(p, q)$ th lower order respectively of an entire function $f \in \mathcal{A}(\mathbb{K})$ where $p, q \in \mathbb{N}$. In order to keep accordance with the definition of logarithmic order we will give a minor modification to the original definition of $(p, q)$-order introduced by Juneja et al. [16,17].

Definition 1. Let $f \in \mathcal{A}(\mathbb{K})$ and $p, q \in \mathbb{N}$. Then the $(p, q)$ th order and $(p, q)$ th lower order of $f$ are respectively defined as:

$$
\begin{aligned}
& \rho^{(p, q)}(f) \\
& \lambda^{(p, q)}(f)
\end{aligned}=\lim _{r \rightarrow+\infty} \sup \frac{\log ^{[p]}|f|(r)}{\log ^{[q]} r} .
$$

These definitions extend the generalized order $\rho^{[l]}(f)$ and generalized lower order $\lambda^{[l]}(f)$ of $f \in \mathcal{A}(\mathbb{K})$ for each integer $l \geq 2$ since these correspond to the particular case $\rho^{[l]}(f)=$ $\rho^{(l, 1)}(f)$ and $\lambda^{[l]}(f)=\lambda^{(l, 1)}(f)$. Clearly $\rho^{(2,1)}(f)=\rho(f)$ and $\lambda^{(2,1)}(f)=\lambda(f)$. The above definition avoid the restriction $p>q$ and give the idea of generalized logarithmic order.

However in this connection we just introduce the following definition which is analogous to the definition of Juneja et al. [16,17].

Definition 2. An entire function $f \in \mathcal{A}(\mathbb{K})$ is said to have index-pair $(p, q)$, where $p$ and $q \in \mathbb{N}$, if $b<\rho^{(p, q)}(f)<\infty$ and $\rho^{(p-1, q-1)}(f)$ is not a nonzero finite number, where $b=1$ if $p=q$ and $b=0$ otherwise. Moreover if $0<\rho^{(p, q)}(f)<\infty$, then

$$
\begin{cases}\rho^{(p-n, q)}(f)=\infty & \text { for } n<p \\ \rho^{(p, q-n)}(f)=0 & \text { for } n<q \\ \rho^{(p+n, q+n)}(f)=1 & \text { for } n=1,2, \ldots\end{cases}
$$

Similarly for $0<\lambda^{(p, q)}(f)<\infty$, one can easily verify that

$$
\begin{cases}\lambda^{(p-n, q)}(f)=\infty & \text { for } n<p, \\ \lambda^{(p, q-n)}(f)=0 & \text { for } n<q, \\ \lambda^{(p+n, q+n)}(f)=1 & \text { for } n=1,2, \ldots\end{cases}
$$

The main aim of this paper is to establish some results related to the growth properties of composite $p$-adic entire functions on the basis of $(p, q)$ th order and $(p, q)$ th lower order, where $p, q \in \mathbb{N}$. 


\section{LEMMA}

In this section we present the following lemma which can be found in [4] or [5] and will be needed in the sequel.

Lemma 1. Let $f, g \in \mathcal{A}(\mathbb{K})$. Then for all sufficiently large values of $r$ the following equality holds

$$
|f \circ g|(r)=|f|(|g|(r)) \text {. }
$$

\section{MAIN RESULTS}

Theorem 1. Let $f, g \in \mathcal{A}(\mathbb{K})$ be such that $\rho^{(m, n)}(g)<\lambda^{(p, q)}(f) \leq \rho^{(p, q)}(f)<\infty$, where $p, q, m, n \in \mathbb{N}$. Then

$$
\text { (i) } \lim _{r \rightarrow+\infty} \frac{\log ^{[p]}|f \circ g|\left(\exp ^{[n-1]} r\right)}{\log ^{[p-1]}|f|\left(\exp ^{[q-1]} r\right)}=0 \quad \text { if } q \geq m
$$

and

$$
\text { (ii) } \lim _{r \rightarrow+\infty} \frac{\log ^{[p+m-q-1]}|f \circ g|\left(\exp ^{[n-1]} r\right)}{\log ^{[p-1]}|f|\left(\exp ^{[q-1]} r\right)}=0 \quad \text { if } q<m \text {. }
$$

Proof. We get from Lemma 1, for all sufficiently large positive numbers of $r$ that

$$
\log ^{[p]}|f \circ g|\left(\exp ^{[n-1]} r\right)=\log [p]|f|\left(|g|\left(\exp ^{[n-1]} r\right)\right)
$$

i.e.,

$$
\log ^{[p]}|f \circ g|\left(\exp ^{[n-1]} r\right) \leqslant\left(\rho^{(p, q)}(f)+\varepsilon\right) \log [q]|g|\left(\exp ^{[n-1]} r\right) .
$$

Now the following two cases may arise.

Case I. Let $q \geqslant m$. Then we have from (1) for all sufficiently large positive numbers of $r$ that

$$
\log [p]|f \circ g|\left(\exp ^{[n-1]} r\right) \leqslant\left(\rho^{(p, q)}(f)+\varepsilon\right) \log [m-1]|g|\left(\exp ^{[n-1]} r\right)
$$

i.e.,

$$
\log ^{[p]}|f \circ g|\left(\exp ^{[n-1]} r\right) \leqslant\left(\rho^{(p, q)}(f)+\varepsilon\right) r^{\left(\rho^{(m, n)}(g)+\varepsilon\right)} .
$$

Case II. Let $q<m$. Then for all sufficiently large positive numbers of $r$ we get from (1) that

$$
\log ^{[p]}|f \circ g|\left(\exp ^{[n-1]} r\right) \leqslant\left(\rho^{(p, q)}(f)+\varepsilon\right) \exp ^{[m-q]} \log { }^{[m]}|g|\left(\exp ^{[n-1]} r\right) .
$$

Further for all sufficiently large positive numbers of $r$, it follows that

$$
\log ^{[m]}|g|\left(\exp ^{[n-1]} r\right) \leqslant \log \left(r^{\rho^{(m, n)}(g)+\varepsilon}\right)
$$

i.e.,

$$
\exp ^{[m-q]} \log { }^{[m]}|g|\left(\exp ^{[n-1]} r\right) \leqslant \exp ^{[m-q-1]}\left(r^{\rho^{(m, n)}(g)+\varepsilon}\right) .
$$

Now from (4) and (5) we have for all sufficiently large positive numbers of $r$ that

$$
\log [p]|f \circ g|\left(\exp ^{[n-1]} r\right) \leq\left(\rho^{(p, q)}(f)+\varepsilon\right) \exp ^{[m-q-1]}\left(r^{\rho^{(m, n)}(g)+\varepsilon}\right)
$$


i.e.,

$$
\log ^{[p+1]}|f \circ g|\left(\exp ^{[n-1]} r\right) \leqslant \exp ^{[m-q-2]}\left(r^{\rho^{(m, n)}(g)+\varepsilon}\right)+O(1)
$$

i.e.,

$$
\log [p+1]|f \circ g|\left(\exp ^{[n-1]} r\right) \leqslant \exp ^{[m-q-2]}\left(r^{\rho^{(m, n)}(g)+\varepsilon}\right)\left(1+\frac{O(1)}{\exp ^{[m-q-2]}\left(r^{\rho^{(m, n)}(g)+\varepsilon}\right)}\right)
$$

i.e.,

$$
\log ^{[p+m-q-1]}|f \circ g|\left(\exp ^{[n-1]} r\right) \leqslant r^{\rho_{g}(m, n)+\varepsilon}\left(1+\frac{O(1)}{\exp ^{[m-q-2]}\left(r^{\rho^{(m, n)}(g)+\varepsilon}\right)}\right) .
$$

Also from the definition of $\lambda^{(p, q)}(f)$, we get for all sufficiently large positive numbers of $r$ that

$$
\log [p-1]|f|\left(\exp ^{[q-1]} r\right) \geqslant r^{\left(\lambda^{(p, q)}(f)-\varepsilon\right)}
$$

Now combining (3) of Case I and (7) we get for all sufficiently large positive numbers of $r$ that

$$
\frac{\log ^{[p]}|f \circ g|\left(\exp ^{[n-1]} r\right)}{\log ^{[p-1]}|f|\left(\exp ^{[q-1]} r\right)} \leq \frac{\left(\rho_{f}(p, q)+\varepsilon\right) r^{\left(\rho^{(m, n)}(g)+\varepsilon\right)}}{r^{\left(\lambda^{(p, q)}(f)-\varepsilon\right)}}
$$

Since $\rho^{(m, n)}(g)<\lambda^{(p, q)}(f)$ we can choose $\varepsilon(>0)$ in such a way that

$$
\rho^{(m, n)}(g)+\varepsilon<\lambda^{(p, q)}(f)-\varepsilon
$$

Therefore in view of (9) it follows from (8) that

$$
\lim _{r \rightarrow+\infty} \frac{\log ^{[p]}|f \circ g|\left(\exp ^{[n-1]} r\right)}{\log ^{[p-1]}|f|\left(\exp ^{[q-1]} r\right)}=0 .
$$

Hence the first part of the theorem follows.

Further combining (6) of Case II and (7) we obtain for all sufficiently large positive numbers of $r$ that

$$
\frac{\log ^{[p+m-q-1]}|f \circ g|\left(\exp ^{[n-1]} r\right)}{\log ^{[p-1]}|f|\left(\exp ^{[q-1]} r\right)} \leq \frac{r^{\rho^{(m, n)}(g)+\varepsilon}\left(1+\frac{O(1)}{\exp ^{[m-q-2]}\left(r^{\left(\rho^{(m, n)}(g)+\varepsilon\right.}\right)}\right)}{r^{\left(\lambda^{(p, q)}(f)-\varepsilon\right)}}
$$

Therefore in view of (9) we get from above that

$$
\lim _{r \rightarrow+\infty} \frac{\log ^{[p+m-q-1]}|f \circ g|\left(\exp ^{[n-1]} r\right)}{\log ^{[p-1]}|f|\left(\exp ^{[q-1]} r\right)}=0 .
$$

Thus the theorem follows.

Theorem 2. Let $f, g \in \mathcal{A}(\mathbb{K})$ be such that $\lambda^{(m, n)}(g)<\lambda^{(p, q)}(f) \leq \rho^{(p, q)}(f)<\infty$, where $p, q, m, n \in \mathbb{N}$. Then

and

$$
\text { (i) } \varliminf_{r \rightarrow+\infty} \frac{\log ^{[p]}|f \circ g|\left(\exp ^{[n-1]} r\right)}{\log ^{[p-1]}|f|\left(\exp ^{[q-1]} r\right)}=0 \quad \text { if } q \geq m
$$

$$
\text { (ii) } \varliminf_{r \rightarrow+\infty} \frac{\log ^{[p+m-q-1]}|f \circ g|\left(\exp ^{[n-1]} r\right)}{\log ^{[p-1]}|f|\left(\exp ^{[q-1]} r\right)}=0 \quad \text { if } q<m \text {. }
$$


The proof of Theorem 2 is omitted as it can be carried out in the line of Theorem 1 .

Theorem 3. Let $f, g \in \mathcal{A}(\mathbb{K})$ be such that $0<\lambda^{(p, q)}(f) \leq \rho^{(p, q)}(f)<\infty$ and $\rho^{(m, n)}(g)<\infty$, where $p, q, m, n \in \mathbb{N}$. Then

$$
\text { (i) } \varlimsup_{r \rightarrow+\infty} \frac{\log [p+1]|f \circ g|\left(\exp ^{[n-1]} r\right)}{\log ^{[p]}|f|\left(\exp ^{[q-1]} r\right)} \leqslant \frac{\rho^{(m, n)}(g)}{\lambda^{(p, q)}(f)} \quad \text { if } q \geq m
$$

and

$$
\text { (ii) } \varlimsup_{r \rightarrow+\infty} \frac{\log ^{[p+m-q]}|f \circ g|\left(\exp ^{[n-1]} r\right)}{\log ^{[p]}|f|\left(\exp ^{[q-1]} r\right)} \leqslant \frac{\rho^{(m, n)}(g)}{\lambda^{(p, q)}(f)} \quad \text { if } q<m \text {. }
$$

Proof. In view of the definition $\lambda^{(p, q)}(f)$, we have for all sufficiently large positive numbers of $r$ that

$$
\log ^{[p]}|f|\left(\exp ^{[q-1]} r\right) \geq\left(\lambda^{(p, q)}(f)-\varepsilon\right) \log r .
$$

Case I. If $q \geqslant m$, then from (3) and (11) we get for all sufficiently large positive numbers of $r$ that

$$
\frac{\log ^{[p+1]}|f \circ g|\left(\exp ^{[n-1]} r\right)}{\log ^{[p]}|f|\left(\exp ^{[q-1]} r\right)} \leqslant \frac{\left(\rho^{(m, n)}(g)+\varepsilon\right) \log r+\log \left(\rho^{(p, q)}(f)+\varepsilon\right)}{\left(\lambda^{(p, q)}(f)-\varepsilon\right) \log r} .
$$

As $\varepsilon(>0)$ is arbitrary, it follows from above that

$$
\varlimsup_{r \rightarrow+\infty} \frac{\log ^{[p+1]}|f \circ g|\left(\exp ^{[n-1]} r\right)}{\log ^{[p]}|f|\left(\exp ^{[q-1]} r\right)} \leqslant \frac{\rho^{(m, n)}(g)}{\lambda^{(p, q)}(f)} .
$$

This proves the first part of the theorem.

Case II. If $q<m$ then from (6) and (11) we obtain for all sufficiently large positive numbers of $r$ that

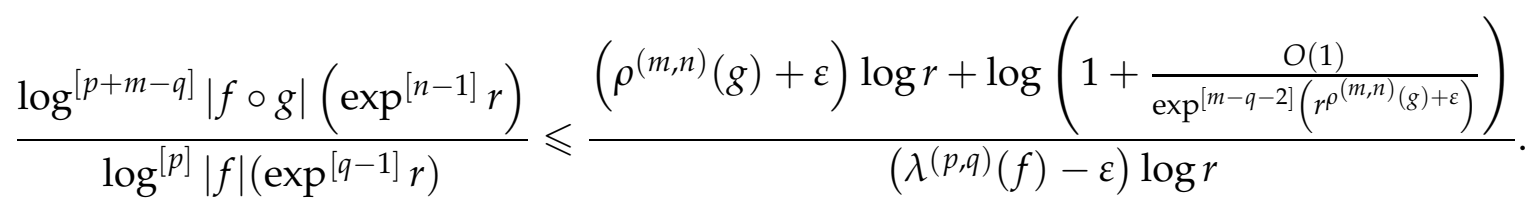

As $\varepsilon(>0)$ is arbitrary, it follows from above that

$$
\varlimsup_{r \rightarrow+\infty} \frac{\log ^{[p+m-q]}|f \circ g|\left(\exp ^{[n-1]} r\right)}{\log ^{[p]}|f|\left(\exp ^{[q-1]} r\right)} \leqslant \frac{\rho^{(m, n)}(g)}{\lambda^{(p, q)}(f)} .
$$

Thus the second part of the theorem is established.

Theorem 4. Let $f, g \in \mathcal{A}(\mathbb{K})$ be such that $0<\lambda^{(p, q)}(f) \leq \rho^{(p, q)}(f)<\infty$ and $\lambda^{(m, n)}(g)>0$, where $p, q, m, n \in \mathbb{N}$. Then for any positive integer $l$, we have

$$
\begin{aligned}
& \text { (i) } \lim _{r \rightarrow \infty} \frac{\log ^{[p]}|f \circ g|\left(\exp ^{[n-1]} r\right)}{\log ^{[p+1]}|f|\left(\exp ^{[l]} r\right)}=\infty \quad \text { if } q<m \text { and } q \geqslant l ; \\
& \text { (ii) } \lim _{r \rightarrow \infty} \frac{\log ^{[p]}|f \circ g|\left(\exp ^{[n-1]} r\right)}{\log ^{[p-q-l+1]}|f|\left(\exp ^{[l]} r\right)}=\infty \quad \text { if } q<m \text { and } q<l ;
\end{aligned}
$$


(iii) $\quad \lim _{r \rightarrow \infty} \frac{\log ^{[p+m-q-1]}|f \circ g|\left(\exp ^{[n-1]} r\right)}{\log ^{[p+1]}|f|\left(\exp ^{[l]} r\right)}=\infty \quad$ if $q>m$ and $q<l$;

and

$$
\text { (iv) } \lim _{r \rightarrow \infty} \frac{\log ^{[p+m-q-1]}|f \circ g|\left(\exp ^{[n-1]} r\right)}{\log ^{[p+1]}|f|\left(\exp ^{[l]} r\right)}=\infty \quad \text { if } q>m \text { and } q \geqslant l \text {. }
$$

Proof. Let us choose $0<\varepsilon<\min \left\{\lambda^{(p, q)}(f), \lambda^{(m, n)}(g)\right\}$. Now for all sufficiently large positive numbers of $r$ we get from Lemma 1,

$$
\log ^{[p]}|f \circ g|\left(\exp ^{[n-1]} r\right) \geqslant\left(\lambda^{(p, q)}(f)-\varepsilon\right) \log [q]|g|\left(\exp ^{[n-1]} r\right) .
$$

Further from the definition of $(m, n)$ th lower order of $g$ we have for all sufficiently large positive numbers of $r$ that

$$
\log { }^{[m]}|g|\left(\exp ^{[n-1]} r\right) \geqslant \log r^{\left(\lambda^{(m, n)}(g)-\varepsilon\right)} .
$$

Now the following two cases may arise.

Case I. Let $q<m$. Then from (12) and (13) we obtain for all sufficiently large positive numbers of $r$ that

$$
\log [p]|f \circ g|\left(\exp ^{[n-1]} r\right) \geqslant\left(\lambda^{(p, q)}(f)-\varepsilon\right) \exp ^{[m-q]} \log { }^{[m]}|g|\left(\exp ^{[n-1]} r\right)
$$

i.e.,

$$
\begin{aligned}
& \log ^{[p]}|f \circ g|\left(\exp ^{[n-1]} r\right) \geqslant\left(\lambda^{(p, q)}(f)-\varepsilon\right) \exp ^{[m-q]} \log r^{\left(\lambda^{(m, n)}(g)-\varepsilon\right)} \\
& \log ^{[p]}|f \circ g|\left(\exp ^{[n-1]} r\right) \geqslant\left(\lambda^{(p, q)}(f)-\varepsilon\right) \exp ^{[m-q-1]} r^{\left(\lambda^{(m, n)}(g)-\varepsilon\right)} .
\end{aligned}
$$

Case II. Let $q>m$. Then from (12) and (13) it follows for all sufficiently large positive numbers of $r$ that

$$
\log ^{[p]}|f \circ g|\left(\exp ^{[n-1]} r\right) \geqslant\left(\lambda^{(p, q)}(f)-\varepsilon\right) \log [q-m] \log r^{\left(\lambda^{(m, n)}(g)-\varepsilon\right)}
$$

i.e.,

$$
\log [p+m-q-1]|f \circ g|\left(\exp ^{[n-1]} r\right) \geqslant r^{\left(\lambda^{(m, n)}(g)-\varepsilon\right)} .
$$

Again from the definition of $\rho^{(p, q)}(f)$ we get for all sufficiently large positive numbers of $r$ that

$$
\log [p]|f|\left(\exp ^{[l]} r\right) \leq\left(\rho^{(p, q)}(f)+\varepsilon\right) \log ^{[q]} \exp ^{[l]} r .
$$

Now the following two cases may arise.

Case III. Let $q \geqslant l$. Then we have from (17) for all sufficiently large positive numbers of $r$ that

$$
\log ^{[p]}|f|\left(\exp ^{[l]} r\right) \leq\left(\rho^{(p, q)}(f)+\varepsilon\right) \log ^{[q-l]} r
$$

i.e.,

$$
\log ^{[p+1]}|f|\left(\exp ^{[l]} r\right) \leq \log { }^{[q-l+1]} r+\log \left(\rho^{(p, q)}(f)+\varepsilon\right) .
$$

Case IV. Let $q<l$. Then we have from (17) for all sufficiently large positive numbers of $r$ that

$$
\log ^{[p]}|f|\left(\exp ^{[l]} r\right) \leq\left(\rho^{(p, q)}(f)+\varepsilon\right) \exp ^{[l-q]} r
$$


i.e.,

i.e.,

$$
\log ^{[p+1]}|f|\left(\exp ^{[l]} r\right) \leq \exp ^{[l-q-1]} r+\log \left(\rho^{(p, q)}(f)+\varepsilon\right)
$$

$$
\log ^{[p-q+l+1]}|f|\left(\exp ^{[l]} r\right) \leq \log r+O(1) .
$$

Now combining (15) of Case I and (18) of Case III it follows for all sufficiently large positive numbers of $r$ that

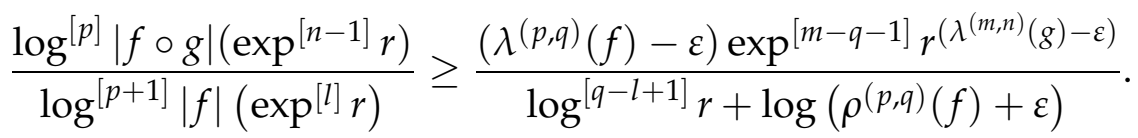

Since $q<m$, we get from the above that

$$
\lim _{r \rightarrow+\infty} \frac{\log ^{[p]}|f \circ g|\left(\exp ^{[n-1]} r\right)}{\log ^{[p+1]}|f|\left(\exp ^{[l]} r\right)}=\infty
$$

This proves the first part of the theorem.

Again in view of (15) of Case I and (19) of Case IV we have for all sufficiently large positive numbers of $r$ that

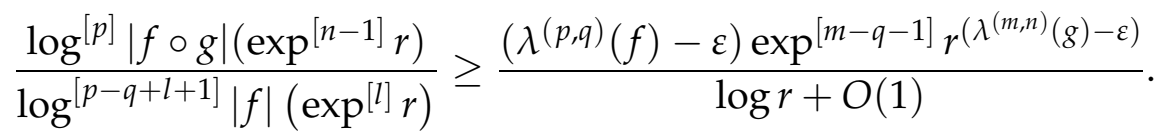

When $q<m$ and $q<l$ then we get from (20) that

$$
\lim _{r \rightarrow+\infty} \frac{\log ^{[p]}|f \circ g|\left(\exp ^{[n-1]} r\right)}{\log ^{[p-q+l+1]}|f|\left(\exp ^{[l]} r\right)}=\infty .
$$

This establishes the second part of the theorem.

Now in view of (16) of Case II and (18) of Case III we get for all sufficiently large positive numbers of $r$ that

$$
\frac{\log ^{[p+m-q-1]}|f \circ g|\left(\exp ^{[n-1]} r\right)}{\log ^{[p+1]}|f|\left(\exp ^{[l]} r\right)} \geq \frac{r^{\left(\lambda^{(m, n)}(g)-\varepsilon\right)}}{\log ^{[q-l+1]} r+\log \left(\rho^{(p, q)}(f)+\varepsilon\right)}
$$

i.e.,

$$
\lim _{r \rightarrow+\infty} \frac{\log ^{[p+m-q-1]}|f \circ g|\left(\exp ^{[n-1]} r\right)}{\log ^{[p+1]}|f|\left(\exp ^{[l]} r\right)}=\infty,
$$

from which the third part of the theorem follows.

Again from (16) of Case II and (19) of Case IV we have for all sufficiently large positive numbers of $r$ that

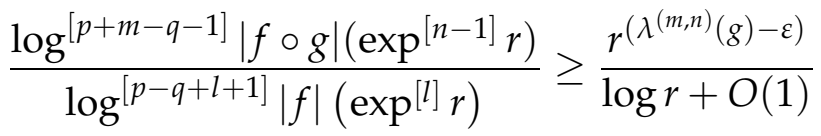

i.e.,

$$
\lim _{r \rightarrow+\infty} \frac{\log ^{[p+m-q-1]}|f \circ g|\left(\exp ^{[n-1]} r\right)}{\log ^{[p-q+l+1]}|f|\left(\exp ^{[l]} r\right)}=\infty .
$$

This proves the fourth part of the theorem. Thus the theorem follows. 
Theorem 5. Let $f, g, h, k \in \mathcal{A}(\mathbb{K})$ be such that $0<\rho^{(a, b)}(h)<\infty, \lambda^{(p, q)}(f)>0, \lambda^{(m, n)}(g)>0$ and $\rho^{(c, d)}(k)<\lambda^{(m, n)}(g)$, where $a, b, c, d, p, q, m, n \in \mathbb{N}$. Then

$$
\begin{aligned}
& \text { (i) } \lim _{r \rightarrow+\infty} \frac{\log ^{[p]}|f \circ g|\left(\exp ^{[n-1]} r\right)}{\log ^{[a]}|h \circ k|(r)}=\infty \quad \text { if } b \geqslant c \text { and } q<m, \\
& \text { (ii) } \lim _{r \rightarrow+\infty} \frac{\log ^{[p]}|f \circ g|\left(\exp ^{[n-1]} r\right)}{\log ^{[a+c-b-1]}|h \circ k|(r)}=\infty \quad \text { if } b<c \text { and } q<m, \\
& \text { (iii) } \lim _{r \rightarrow+\infty} \frac{\log ^{[p+m-q-1]}|f \circ g|\left(\exp ^{[n-1]} r\right)}{\log ^{[a]}|h \circ k|(r)}=\infty \quad \text { if } b \geqslant c \text { and } q \geqslant m, \\
& \text { and (iv) } \lim _{r \rightarrow+\infty} \frac{\log ^{[p+m-q-1]}|f \circ g|\left(\exp ^{[n-1]} r\right)}{\log ^{[a+c-b-1]}|h \circ k|(r)}=\infty \quad \text { if } b<c \text { and } q \geqslant m \text {. }
\end{aligned}
$$

Proof. In view of Lemma 1 we obtain for all sufficiently large positive numbers of $r$ that

$$
\log ^{[a]}|h \circ k|(r) \leqslant\left(\rho^{(a, b)}(h)+\varepsilon\right) \log ^{[b]}|k|(r) .
$$

Now from the definition of $(c, d)$ th order of $k$ we get for arbitrary positive $\varepsilon$ and for all sufficiently large positive numbers of $r$ that

$$
\log [c]|k|(r) \leqslant\left(\rho^{(c, d)}(k)+\varepsilon\right) \log ^{[d]} r
$$

i.e.,

$$
\log [c]|k|(r) \leqslant\left(\rho^{(c, d)}(k)+\varepsilon\right) \log r
$$

i.e.,

$$
\log ^{[c-1]}|k|(r) \leqslant r^{\left(\rho^{(c, d)}(k)+\varepsilon\right)} .
$$

Now the following cases may arise.

Case I. Let $b \geqslant c$. Then we have from (21) for all sufficiently large positive numbers of $r$ that

$$
\log ^{[a]}|h \circ k|(r) \leqslant\left(\rho^{(a, b)}(h)+\varepsilon\right) \log ^{[c-1]}|k|(r) .
$$

So from (23) and (24), it follows for all sufficiently large positive numbers of $r$ that

$$
\log ^{[a]}|h \circ k|(r) \leqslant\left(\rho^{(a, b)}(h)+\varepsilon\right) r^{\left(\rho^{(c, d)}(k)+\varepsilon\right)} .
$$

Case II. Let $b<c$. Then we get from (21) for all sufficiently large positive numbers of $r$ that

$$
\log ^{[a]}|h \circ k|(r) \leqslant\left(\rho^{(a, b)}(h)+\varepsilon\right) \exp ^{[c-b]} \log ^{[c]}|k|(r) .
$$

Now from (22) and (26) we obtain for all sufficiently large positive numbers of $r$ that

$$
\log ^{[a]}|h \circ k|(r) \leqslant\left(\rho^{(a, b)}(h)+\varepsilon\right) \exp ^{[c-b]} \log r^{\left(\rho^{(c, d)}(k)+\varepsilon\right)}
$$

i.e.,

$$
\log ^{[a+c-b-1]}|h \circ k|(r) \leqslant r^{\left(\rho^{(c, d)}(k)+\varepsilon\right)}+O(1) .
$$


Since $\rho^{(c, d)}(k)<\lambda^{(m, n)}(g)$ we can choose $\varepsilon(>0)$ in such a way that

$$
\rho^{(c, d)}(k)+\varepsilon<\lambda^{(m, n)}(g)-\varepsilon .
$$

Now combining (25) of Case I, (15) and in view of (28) it follows for all sufficiently large positive numbers of $r$ that

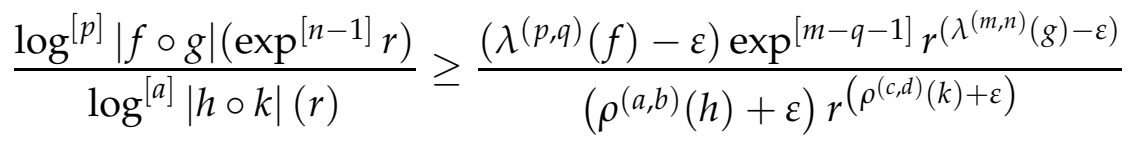

i.e.,

$$
\lim _{r \rightarrow+\infty} \frac{\log [p]|f \circ g|\left(\exp ^{[n-1]} r\right)}{\log ^{[a]}|h \circ k|(r)}=\infty,
$$

from which the first part of the theorem follows.

Again combining (27) of Case II, (15) and in view of (28) we obtain for all sufficiently large positive numbers of $r$ that

$$
\frac{\log ^{[p]}|f \circ g|\left(\exp ^{[n-1]} r\right)}{\log ^{[a+c-b-1]}|h \circ k|(r)} \geq \frac{\left(\lambda^{(p, q)}(f)-\varepsilon\right) \exp ^{[m-q-1]} r^{\left(\lambda^{(m, n)}(g)-\varepsilon\right)}}{r^{\left(\rho^{(c, d)}(k)+\varepsilon\right)}+O(1)}
$$

i.e.,

$$
\lim _{r \rightarrow+\infty} \frac{\log ^{[p]}|f \circ g|\left(\exp ^{[n-1]} r\right)}{\log ^{[a+c-b-1]}|h \circ k|(r)}=\infty .
$$

This establishes the second part of the theorem.

Further in view of (25) of Case I and (16) we get for all sufficiently large positive numbers of $r$ that

$$
\frac{\log ^{[p+m-q-1]}|f \circ g|\left(\exp ^{[n-1]} r\right)}{\log ^{[a]}|h \circ k|(r)} \geq \frac{r^{\left(\lambda^{(m, n)}(g)-\varepsilon\right)}}{\left(\rho^{(a, b)}(h)+\varepsilon\right) r^{\left(\rho^{(c, d)}(k)+\varepsilon\right)}} .
$$

So from (28) and (29) we obtain that

$$
\lim _{r \rightarrow+\infty} \frac{\log ^{[p+m-q-1]}|f \circ g|\left(\exp ^{[n-1]} r\right)}{\log ^{[a]}|h \circ k|(r)}=\infty,
$$

from which the third part of the theorem follows.

Again combining (27) of Case II and (16) it follows for all sufficiently large positive numbers of $r$ that

$$
\frac{\log ^{[p+m-q-1]}|f \circ g|\left(\exp ^{[n-1]} r\right)}{\log ^{[a+c-b-1]}|h \circ k|(r)} \geq \frac{r^{\left(\lambda^{(m, n)}(g)-\varepsilon\right)}}{r^{\left(\rho^{(c, d)}(k)+\varepsilon\right)}+O(1)} .
$$

Now in view of (28) we obtain from (30) that

$$
\lim _{r \rightarrow+\infty} \frac{\log ^{[p+m-q-1]}|f \circ g|\left(\exp ^{[n-1]} r\right)}{\log ^{[a+c-b-1]}|h \circ k|(r)}=\infty .
$$

This proves the fourth part of the theorem. Thus the theorem follows. 
Theorem 6. Let $f, g \in \mathcal{A}(\mathbb{K})$ be such that $\rho^{(a, b)}(f \circ g)<\infty$ and $\lambda^{(m, n)}(g)>0$, where $a, b, m, n \in \mathbb{N}$. Then

$$
\lim _{r \rightarrow+\infty} \frac{\left[\log ^{[a]}|f \circ g|\left(\exp ^{[b-1]} r\right)\right]^{2}}{\log ^{[m-1]}|g|\left(\exp ^{[n]} r\right) \cdot \log ^{[m]}|g|\left(\exp ^{[n-1]} r\right)}=0 .
$$

Proof. For any $\varepsilon>0$ we have $\log ^{[a]}|f \circ g|\left(\exp ^{[b-1]} r\right) \leq\left(\rho^{(a, b)}(f \circ g)+\varepsilon\right) \log ^{[b]} \exp ^{[b-1]} r$, i.e.,

$$
\log ^{[a]}|f \circ g|\left(\exp ^{[b-1]} r\right) \leq\left(\rho^{(a, b)}(f \circ g)+\varepsilon\right) \log r .
$$

Again we obtain that $\log ^{[m]}|g|\left(\exp ^{[n-1]} r\right) \geq\left(\lambda^{(m, n)}(g)-\varepsilon\right) \log ^{[n]} \exp ^{[n-1]} r$, i.e.,

$$
\log ^{[m]}|g|\left(\exp ^{[n-1]} r\right) \geq\left(\lambda^{(m, n)}(g)-\varepsilon\right) \log r .
$$

Similarly we have $\log ^{[m]}|g|\left(\exp ^{[n]} r\right) \geq\left(\lambda^{(m, n)}(g)-\varepsilon\right) \log ^{[n]} \exp ^{[n]} r$, i.e.,

$$
\log { }^{[m-1]}|g|\left(\exp ^{[n]} r\right) \geq \exp \left[\left(\lambda^{(m, n)}(g)-\varepsilon\right) r\right] .
$$

From (31) and (32) we have for all sufficiently large positive numbers of $r$ that

$$
\frac{\log ^{[a]}|f \circ g|\left(\exp ^{[b-1]} r\right)}{\log ^{[m]}|g|\left(\exp ^{[n-1]} r\right)} \leq \frac{\left(\rho^{(a, b)}(f \circ g)+\varepsilon\right) \log r}{\left(\lambda^{(m, n)}(g)-\varepsilon\right) \log r} .
$$

As $\varepsilon(>0)$ is arbitrary we obtain from the above that

$$
\varlimsup_{r \rightarrow+\infty} \frac{\log ^{[a]}|f \circ g|\left(\exp ^{[b-1]} r\right)}{\log ^{[m]}|g|\left(\exp ^{[n-1]} r\right)} \leq \frac{\rho^{(a, b)}(f \circ g)}{\lambda^{(m, n)}(g)} .
$$

Again from (31) and (33) we get for all sufficiently large positive numbers of $r$ that

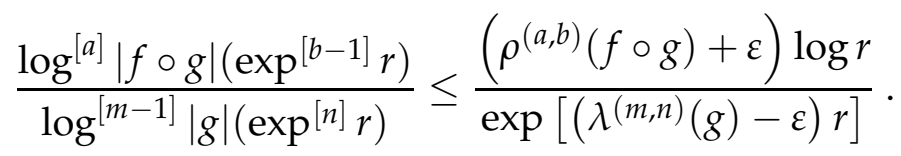

Since $\varepsilon(>0)$ is arbitrary it follows from the above that

$$
\lim _{r \rightarrow+\infty} \frac{\log ^{[a]}|f \circ g|\left(\exp ^{[b-1]} r\right)}{\log ^{[m-1]}|g|\left(\exp ^{[n]} r\right)}=0 .
$$

Thus the theorem follows from (34) and (35).

Theorem 7. Let $f, g \in \mathcal{A}(\mathbb{K})$ be such that $0<\lambda^{(p, q)}(f) \leq \rho^{(p, q)}(f)<\infty$ and $0<\lambda^{(m, n)}(g) \leq$ $\rho^{(m, n)}(g)<\infty$, where $p, q, m, n \in \mathbb{N}$. Then

$$
\begin{aligned}
& \text { (i) } \frac{\lambda^{(p, q)}(f) \cdot \lambda^{(m, n)}(g)}{\rho^{(p, q)}(f)} \leq \varliminf_{r \rightarrow+\infty} \frac{\log [p]|f \circ g|(r)}{\log ^{[p]}|f|(r)} \leqslant \min \left\{\rho^{(m, n)}(g), \frac{\rho^{(p, q)}(f) \cdot \lambda^{(m, n)}(g)}{\lambda^{(p, q)}(f)}\right\} \text {; }
\end{aligned}
$$

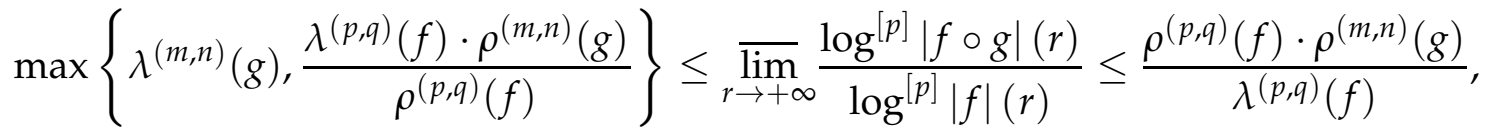

when $q=m=n$, 
(ii) $\frac{\lambda^{(p, q)}(f) \cdot \lambda^{(m, n)}(g)}{\rho^{(p, q)}(f)} \leq \underset{r \rightarrow+\infty}{\lim } \frac{\log ^{[p]}|f \circ g|(r)}{\log ^{[p]}|f|\left(\exp ^{[q-n]} r\right)} \leqslant \min \left\{\rho^{(m, n)}(g), \frac{\rho^{(p, q)}(f) \cdot \lambda^{(m, n)}(g)}{\lambda^{(p, q)}(f)}\right\}$; $\max \left\{\lambda^{(m, n)}(g), \frac{\lambda^{(p, q)}(f) \cdot \rho^{(m, n)}(g)}{\rho^{(p, q)}(f)}\right\} \leq \varlimsup_{r \rightarrow+\infty} \frac{\log [p]|f \circ g|(r)}{\log ^{[p]}|f|\left(\exp ^{[q-n]} r\right)} \leq \frac{\rho^{(p, q)}(f) \cdot \rho^{(m, n)}(g)}{\lambda^{(p, q)}(f)}$, when $q=m>$ or $<n$,

$$
\begin{aligned}
& \frac{\lambda^{(p, q)}(f)}{\rho^{(p, q)}(f)} \leq \varliminf_{r \rightarrow+\infty} \frac{\log [p]}{\log ^{[p]}|f|\left(\exp ^{[m-n]} r\right)} \leqslant \min \left\{1, \frac{\rho^{(p, q)}(f)}{\lambda^{(p, q)}(f)}\right\} ; \\
& \max \left\{1, \frac{\lambda^{(p, q)}(f)}{\rho^{(p, q)}(f)}\right\} \leq \varlimsup_{r \rightarrow+\infty} \frac{\log ^{[p]}|f \circ g|(r)}{\log ^{[p]}|f|\left(\exp ^{[m-n]} r\right)} \leq \frac{\rho^{(p, q)}(f)}{\lambda^{(p, q)}(f)}
\end{aligned}
$$

when $q>m$,

$$
\begin{aligned}
\frac{\lambda^{(m, n)}(g)}{\rho^{(p, q)}(f)} & \leq \varliminf_{r \rightarrow+\infty} \frac{\log ^{[p+m-q]}|f \circ g|(r)}{\log ^{[p]}|f|(r)} \leqslant \min \left\{\frac{\lambda^{(m, n)}(g)}{\lambda^{(p, q)}(f)}, \frac{\rho^{(m, n)}(g)}{\rho^{(p, q)}(f)}\right\} \\
& \leq \max \left\{\frac{\lambda^{(m, n)}(g)}{\lambda^{(p, q)}(f)}, \frac{\rho^{(m, n)}(g)}{\rho^{(p, q)}(f)}\right\} \leq \varlimsup_{r \rightarrow+\infty} \frac{\log ^{[p+m-q]}|f \circ g|(r)}{\log ^{[p]}|f|(r)} \leq \frac{\rho^{(m, n)}(g)}{\lambda^{(p, q)}(f)}
\end{aligned}
$$

when $m>q=n$,

$$
\text { (v) } \begin{aligned}
\frac{\lambda^{(m, n)}(g)}{\rho^{(p, q)}(f)} & \leq \underset{r \rightarrow+\infty}{\lim _{\log [p]}|f|\left(\exp ^{[q-n]} r\right)} \leqslant \min \left\{\frac{\lambda^{(m, n)}(g)}{\lambda^{(p, q)}(f)}, \frac{\rho^{(m, n)}(g)}{\rho^{(p, q)}(f)}\right\} \\
& \leq \max \left\{\frac{\lambda^{(m, n)}(g)}{\lambda^{(p, q)}(f)}, \frac{\rho^{(m, n)}(g)}{\rho^{(p, q)}(f)}\right\} \leq \varlimsup_{r \rightarrow+\infty} \frac{\log ^{[p+m-q]}|f \circ g|(r)}{\log ^{[p]}|f|\left(\exp ^{[q-n]} r\right)} \leq \frac{\rho^{(m, n)}(g)}{\lambda^{(p, q)}(f)},
\end{aligned}
$$

when $m>q>n$, and

$$
\begin{aligned}
& \frac{\lambda^{(m, n)}(g)}{\rho^{(p, q)}(f)} \leq \varliminf_{r \rightarrow+\infty} \frac{\log ^{[p+m-q]}|f \circ g|\left(\exp ^{[n-q]} r\right)}{\log ^{[p]}|f|\left(\exp ^{[q-n]} r\right)} \leqslant \min \left\{\frac{\lambda^{(m, n)}(g)}{\lambda^{(p, q)}(f)}, \frac{\rho^{(m, n)}(g)}{\rho^{(p, q)}(f)}\right\} \\
& \leq \max \left\{\frac{\lambda^{(m, n)}(g)}{\lambda^{(p, q)}(f)}, \frac{\rho^{(m, n)}(g)}{\rho^{(p, q)}(f)}\right\} \leq \varlimsup_{r \rightarrow+\infty} \frac{\log ^{[p+m-q]}|f \circ g|\left(\exp ^{[n-q]} r\right)}{\log ^{[p]}|f|\left(\exp ^{[q-n]} r\right)} \\
& \leq \frac{\rho^{(m, n)}(g)}{\lambda^{(p, q)}(f)},
\end{aligned}
$$

when $m>q<n$.

Proof. From the definitions of $(p, q)$ th order and $(p, q)$ th lower order of $f$, we have for all sufficiently large positive numbers of $r$ that

$$
\begin{aligned}
& \log ^{[p]}|f| \leq\left(\rho^{(p, q)}(f)+\varepsilon\right) \log ^{[q]} r, \\
& \log ^{[p]}|f| \geq\left(\lambda^{(p, q)}(f)-\varepsilon\right) \log ^{[q]} r
\end{aligned}
$$

and also for a sequence of positive numbers of $r$ tending to infinity we get that

$$
\begin{aligned}
\log ^{[p]}|f| & \geq\left(\rho^{(p, q)}(f)-\varepsilon\right) \log ^{[q]} r, \\
\log ^{[p]}|f| & \leq\left(\lambda^{(p, q)}(f)+\varepsilon\right) \log ^{[q]} r .
\end{aligned}
$$


Now in view of Lemma 1, we have for all sufficiently large positive numbers of $r$ that

$$
\log ^{[p]}|f \circ g|(r) \leqslant\left(\rho^{(p, q)}(f)+\varepsilon\right) \log ^{[q]}|g|(r)
$$

and also we get for a sequence of positive numbers of $r$ tending to infinity that

$$
\log ^{[p]}|f \circ g|(r) \leqslant\left(\lambda^{(p, q)}(f)+\varepsilon\right) \log ^{[q]}|g|(r) .
$$

Similarly, in view of Lemma 1, it follows for all sufficiently large positive numbers of $r$ that

$$
\log ^{[p]}|f \circ g|(r) \geq\left(\lambda^{(p, q)}(f)-\varepsilon\right) \log ^{[q]}|g|(r)
$$

and also we obtain for a sequence of positive numbers of $r$ tending to infinity that

$$
\log ^{[p]}|f \circ g|(r) \geq\left(\rho^{(p, q)}(f)-\varepsilon\right) \log [q]|g|(r) .
$$

Now the following two cases may arise.

Case I. Let $q=m=n$. Then we have from (40) for all sufficiently large positive numbers of $r$ that

$$
\log ^{[p]}|f \circ g|(r) \leqslant\left(\rho^{(p, q)}(f)+\varepsilon\right)\left(\rho^{(m, n)}(g)+\varepsilon\right) \log ^{[n]} r,
$$

and for a sequence of positive numbers of $r$ tending to infinity that

$$
\log ^{[p]}|f \circ g|(r) \leqslant\left(\rho^{(p, q)}(f)+\varepsilon\right)\left(\lambda^{(m, n)}(g)+\varepsilon\right) \log ^{[n]} r .
$$

Also we obtain from (41) for a sequence of positive numbers of $r$ tending to infinity that

$$
\log ^{[p]}|f \circ g|(r) \leqslant\left(\lambda^{(p, q)}(f)+\varepsilon\right)\left(\rho^{(m, n)}(g)+\varepsilon\right) \log ^{[n]} r .
$$

Further it follows from (42) for all sufficiently large positive numbers of $r$ that

$$
\log ^{[p]}|f \circ g|(r) \geq\left(\lambda^{(p, q)}(f)-\varepsilon\right)\left(\lambda^{(m, n)}(g)-\varepsilon\right) \log ^{[n]} r,
$$

and for a sequence of positive numbers of $r$ tending to infinity that

$$
\log ^{[p]}|f \circ g|(r) \geq\left(\lambda^{(p, q)}(f)-\varepsilon\right)\left(\rho^{(m, n)}(g)-\varepsilon\right) \log ^{[n]} r .
$$

Moreover, we obtain from (43) for a sequence of positive numbers of $r$ tending to infinity that

$$
\log ^{[p]}|f \circ g|(r) \geq\left(\rho^{(p, q)}(f)-\varepsilon\right)\left(\lambda^{(m, n)}(g)-\varepsilon\right) \log ^{[n]} r .
$$

Therefore from (37) and (44), we have for all sufficiently large positive numbers of $r$ that

$$
\begin{aligned}
\frac{\log ^{[p]}|f \circ g|(r)}{\log ^{[p]}|f|(r)} & \leqslant \frac{\left(\rho^{(p, q)}(f)+\varepsilon\right)\left(\rho^{(m, n)}(g)+\varepsilon\right) \log ^{[n]} r}{\left(\lambda^{(p, q)}(f)-\varepsilon\right) \log ^{[q]} r} \\
& =\frac{\left(\rho^{(p, q)}(f)+\varepsilon\right)\left(\rho^{(m, n)}(g)+\varepsilon\right) \log ^{[q]} r}{\left(\lambda^{(p, q)}(f)-\varepsilon\right) \log ^{[q]} r}
\end{aligned}
$$


i.e.,

$$
\varlimsup_{r \rightarrow+\infty} \frac{\log ^{[p]}|f \circ g|(r)}{\log ^{[p]}|f|(r)} \leqslant \frac{\rho^{(p, q)}(f) \cdot \rho^{(m, n)}(g)}{\lambda^{(p, q)}(f)} .
$$

Similarly from (38) and (44), for a sequence of positive numbers of $r$ tending to infinity it follows that

$$
\begin{aligned}
& \frac{\log ^{[p]}|f \circ g|(r)}{\log [p]}|f|(r) \leqslant \frac{\left(\rho^{(p, q)}(f)+\varepsilon\right)\left(\rho^{(m, n)}(g)+\varepsilon\right) \log ^{[n]} r}{\left(\rho^{(p, q)}(f)-\varepsilon\right) \log ^{[q]} r} \\
&=\frac{\left(\rho^{(p, q)}(f)+\varepsilon\right)\left(\rho^{(m, n)}(g)+\varepsilon\right) \log ^{[q]} r}{\left(\rho^{(p, q)}(f)-\varepsilon\right) \log ^{[q]} r}, \\
& \varliminf_{r \rightarrow+\infty} \frac{\log ^{[p]}|f \circ g|(r)}{\log ^{[p]}|f|(r)} \leqslant \rho^{(m, n)}(g) .
\end{aligned}
$$

Also from (37) and (45), we obtain for a sequence of positive numbers of $r$ tending to infinity that

$$
\begin{aligned}
& \frac{\log ^{[p]}|f \circ g|(r)}{\log ^{[p]}|f|(r)} \leqslant \frac{\left(\rho^{(p, q)}(f)+\varepsilon\right)\left(\lambda^{(m, n)}(g)+\varepsilon\right) \log ^{[n]} r}{\left(\lambda^{(p, q)}(f)-\varepsilon\right) \log ^{[q]} r} \\
& =\frac{\left(\rho^{(p, q)}(f)+\varepsilon\right)\left(\lambda^{(m, n)}(g)+\varepsilon\right) \log ^{[q]} r}{\left(\lambda^{(p, q)}(f)-\varepsilon\right) \log ^{[q]} r} \\
& \varliminf_{r \rightarrow+\infty} \frac{\log [p]|f \circ g|(r)}{\log ^{[p]}|f|(r)} \leqslant \frac{\rho^{(p, q)}(f) \cdot \lambda^{(m, n)}(g)}{\lambda^{(p, q)}(f)},
\end{aligned}
$$

Further from (37) and (46), for a sequence of positive numbers of $r$ tending to infinity we have that

$$
\begin{aligned}
& \frac{\log ^{[p]}|f \circ g|(r)}{\log ^{[p]}|f|(r)} \leqslant \frac{\left(\lambda^{(p, q)}(f)+\varepsilon\right)\left(\rho^{(m, n)}(g)+\varepsilon\right) \log ^{[n]} r}{\left(\lambda^{(p, q)}(f)-\varepsilon\right) \log ^{[q]} r} \\
&=\frac{\left(\lambda^{(p, q)}(f)+\varepsilon\right)\left(\rho^{(m, n)}(g)+\varepsilon\right) \log ^{[q]} r}{\left(\lambda^{(p, q)}(f)-\varepsilon\right) \log ^{[q]} r} \\
& \underset{r \rightarrow+\infty}{\underline{\lim }} \frac{\log ^{[p]}|f \circ g|(r)}{\log ^{[p]}|f|(r)} \leqslant \rho^{(m, n)}(g) .
\end{aligned}
$$

Thus from (51), (52) and (53) it follows that

$$
\varliminf_{r \rightarrow+\infty} \frac{\log ^{[p]}|f \circ g|(r)}{\log [p]|f|(r)} \leqslant \min \left\{\rho^{(m, n)}(g), \frac{\rho^{(p, q)}(f) \cdot \lambda^{(m, n)}(g)}{\lambda^{(p, q)}(f)}\right\} .
$$

Further from (36) and (47), for all sufficiently large positive numbers of $r$ we have that

$$
\frac{\log ^{[p]}|f \circ g|(r)}{\log ^{[p]}|f|(r)} \geq \frac{\left(\lambda^{(p, q)}(f)-\varepsilon\right)\left(\lambda^{(m, n)}(g)-\varepsilon\right) \log ^{[n]} r}{\left(\rho^{(p, q)}(f)+\varepsilon\right) \log ^{[q]} r},
$$


i.e.,

$$
\varliminf_{r \rightarrow+\infty} \frac{\log ^{[p]}|f \circ g|(r)}{\log ^{[p]}|f|(r)} \geq \frac{\lambda^{(p, q)}(f) \cdot \lambda^{(m, n)}(g)}{\rho^{(p, q)}(f)} .
$$

Similarly, from (39) and (47) we obtain that

$$
\varlimsup_{r \rightarrow+\infty} \frac{\log ^{[p]}|f \circ g|(r)}{\log ^{[p]}|f|(r)} \geq \lambda^{(m, n)}(g) .
$$

Also from (36) and (48), for a sequence of positive numbers of $r$ tending to infinity we obtain that

$$
\frac{\log ^{[p]}|f \circ g|(r)}{\log ^{[p]}|f|(r)} \geq \frac{\left(\lambda^{(p, q)}(f)-\varepsilon\right)\left(\rho^{(m, n)}(g)-\varepsilon\right) \log ^{[n]} r}{\left(\rho^{(p, q)}(f)+\varepsilon\right) \log ^{[q]} r}
$$

i.e.,

$$
\varlimsup_{r \rightarrow+\infty} \frac{\log ^{[p]}|f \circ g|(r)}{\log ^{[p]}|f|(r)} \geq \frac{\lambda^{(p, q)}(f) \cdot \rho^{(m, n)}(g)}{\rho^{(p, q)}(f)},
$$

and from (36) and (49), for a sequence of positive numbers of $r$ tending to infinity we have that

$$
\frac{\log ^{[p]}|f \circ g|(r)}{\log ^{[p]}|f|(r)} \geq \frac{\left(\rho^{(p, q)}(f)-\varepsilon\right)\left(\lambda^{(m, n)}(g)-\varepsilon\right) \log ^{[n]} r}{\left(\rho^{(p, q)}(f)+\varepsilon\right) \log ^{[q]} r}
$$

i.e.,

$$
\varlimsup_{r \rightarrow+\infty} \frac{\log ^{[p]}|f \circ g|(r)}{\log ^{[p]}|f|(r)} \geq \lambda^{(m, n)}(g) .
$$

Thus from (56), (57) and (58) it follows that

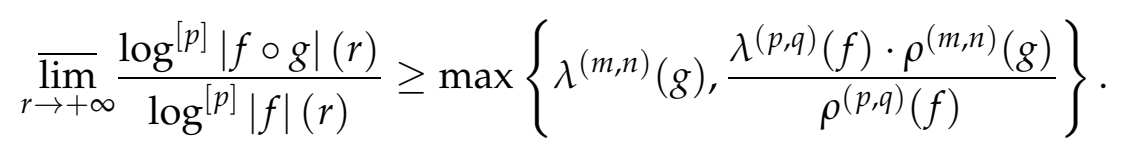

Therefore the first part of the theorem follows from (50), (54), (55) and (59).

Case II. Let $q=m$ and $m>n$ or $n<m$. Now from (37) and (44), for all sufficiently large positive numbers of $r$ we have that

$$
\frac{\log ^{[p]}|f \circ g|(r)}{\log ^{[p]}|f|\left(\exp ^{[q-n]} r\right)} \leqslant \frac{\left(\rho^{(p, q)}(f)+\varepsilon\right)\left(\rho^{(m, n)}(g)+\varepsilon\right) \log ^{[n]} r}{\left(\lambda^{(p, q)}(f)-\varepsilon\right) \log ^{[n]} r}
$$

i.e.,

$$
\varlimsup_{r \rightarrow+\infty} \frac{\log ^{[p]}|f \circ g|(r)}{\log ^{[p]}|f|\left(\exp ^{[q-n]} r\right)} \leqslant \frac{\rho^{(p, q)}(f) \cdot \rho^{(m, n)}(g)}{\lambda^{(p, q)}(f)} .
$$

Similarly, from (38) and (44) for a sequence of positive numbers of $r$ tending to infinity it follows that

$$
\frac{\log ^{[p]}|f \circ g|(r)}{\log ^{[p]}|f|\left(\exp ^{[q-n]} r\right)} \leqslant \frac{\left(\rho^{(p, q)}(f)+\varepsilon\right)\left(\rho^{(m, n)}(g)+\varepsilon\right) \log ^{[n]} r}{\left(\rho^{(p, q)}(f)-\varepsilon\right) \log ^{[n]} r}
$$


i.e.,

$$
\varliminf_{r \rightarrow+\infty} \frac{\log ^{[p]}|f \circ g|(r)}{\log ^{[p]}|f|\left(\exp ^{[q-n]} r\right)} \leqslant \rho^{(m, n)}(g) .
$$

Also from (37) and (45), for a sequence of positive numbers of $r$ tending to infinity we obtain that

$$
\frac{\log ^{[p]}|f \circ g|(r)}{\log ^{[p]}|f|\left(\exp ^{[q-n]} r\right)} \leqslant \frac{\left(\rho^{(p, q)}(f)+\varepsilon\right)\left(\lambda^{(m, n)}(g)+\varepsilon\right) \log ^{[n]} r}{\left(\lambda^{(p, q)}(f)-\varepsilon\right) \log ^{[n]} r}
$$

i.e.,

$$
\varliminf_{r \rightarrow+\infty} \frac{\log ^{[p]}|f \circ g|(r)}{\log ^{[p]}|f|\left(\exp ^{[q-n]} r\right)} \leqslant \frac{\rho^{(p, q)}(f) \cdot \lambda^{(m, n)}(g)}{\lambda^{(p, q)}(f)},
$$

and from (37) and (46), for a sequence of positive numbers of $r$ tending to infinity we have that

$$
\frac{\log ^{[p]}|f \circ g|(r)}{\log ^{[p]}|f|\left(\exp ^{[q-n]} r\right)} \leqslant \frac{\left(\lambda^{(p, q)}(f)+\varepsilon\right)\left(\rho^{(m, n)}(g)+\varepsilon\right) \log ^{[n]} r}{\left(\lambda^{(p, q)}(f)-\varepsilon\right) \log ^{[n]} r}
$$

i.e.,

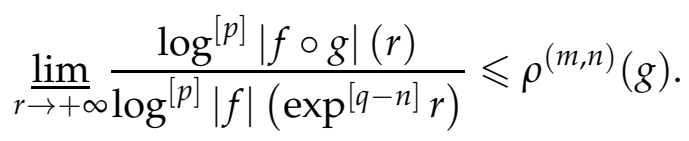

Thus from (61), (62) and (63) it follows that

$$
\varliminf_{r \rightarrow+\infty} \frac{\log ^{[p]}|f \circ g|(r)}{\log ^{[p]}|f|(\exp [q-n] r)} \leqslant \min \left\{\rho^{(m, n)}(g), \frac{\rho^{(p, q)}(f) \cdot \lambda^{(m, n)}(g)}{\lambda^{(p, q)}(f)}\right\} .
$$

Further from (36) and (47), for all sufficiently large positive numbers of $r$ we have that

$$
\frac{\log ^{[p]}|f \circ g|(r)}{\log ^{[p]}|f|\left(\exp ^{[q-n]} r\right)} \geq \frac{\left(\lambda^{(p, q)}(f)-\varepsilon\right)\left(\lambda^{(m, n)}(g)-\varepsilon\right) \log ^{[n]} r}{\left(\rho^{(p, q)}(f)+\varepsilon\right) \log ^{[n]} r}
$$

i.e.,

$$
\varliminf_{r \rightarrow+\infty} \frac{\log ^{[p]}|f \circ g|(r)}{\log ^{[p]}|f|\left(\exp ^{[q-n]} r\right)} \geq \frac{\lambda^{(p, q)}(f) \cdot \lambda^{(m, n)}(g)}{\rho^{(p, q)}(f)} .
$$

Similarly, from (39) and (47) for a sequence of positive numbers of $r$ tending to infinity it follows that

$$
\frac{\log ^{[p]}|f \circ g|(r)}{\log ^{[p]}|f|\left(\exp ^{[q-n]} r\right)} \geq \frac{\left(\lambda^{(p, q)}(f)-\varepsilon\right)\left(\lambda^{(m, n)}(g)-\varepsilon\right) \log ^{[n]} r}{\left(\lambda^{(p, q)}(f)+\varepsilon\right) \log ^{[n]} r}
$$

i.e.,

$$
\varlimsup_{r \rightarrow+\infty} \frac{\log ^{[p]}|f \circ g|(r)}{\log ^{[p]}|f|\left(\exp ^{[q-n]} r\right)} \geq \lambda^{(m, n)}(g) .
$$

Also from (36) and (48), for a sequence of positive numbers of $r$ tending to infinity we obtain that

$$
\frac{\log ^{[p]}|f \circ g|(r)}{\log ^{[p]}|f|\left(\exp ^{[q-n]} r\right)} \geq \frac{\left(\lambda^{(p, q)}(f)-\varepsilon\right)\left(\rho^{(m, n)}(g)-\varepsilon\right) \log ^{[n]} r}{\left(\rho^{(p, q)}(f)+\varepsilon\right) \log ^{[n]} r}
$$


i.e.,

$$
\varlimsup_{r \rightarrow+\infty} \frac{\log ^{[p]}|f \circ g|(r)}{\log ^{[p]}|f|\left(\exp ^{[q-n]} r\right)} \geq \frac{\lambda^{(p, q)}(f) \cdot \rho^{(m, n)}(g)}{\rho^{(p, q)}(f)} .
$$

Similarly from (36) and (49), we get that

$$
\varlimsup_{r \rightarrow+\infty} \frac{\log ^{[p]}|f \circ g|(r)}{\log ^{[p]}|f|\left(\exp ^{[q-n]} r\right)} \geq \lambda^{(m, n)}(g) .
$$

Thus from (66), (67) and (68) it follows that

$$
\varlimsup_{r \rightarrow+\infty} \frac{\log ^{[p]}|f \circ g|(r)}{\log ^{[p]}|f|\left(\exp ^{[q-n]} r\right)} \geq \max \left\{\lambda^{(m, n)}(g), \frac{\lambda^{(p, q)}(f) \cdot \rho^{(m, n)}(g)}{\rho^{(p, q)}(f)}\right\} .
$$

Thus the second part of the theorem follows from (60), (64), (65) and (69).

Case III. Let $q>m$. Then from (40) for all sufficiently large positive numbers of $r$ we have

$$
\log ^{[p]}|f \circ g|(r) \leqslant\left(\rho^{(p, q)}(f)+\varepsilon\right) \log [q-m]\left[\left(\rho^{(m, n)}(g)+\varepsilon\right) \log ^{[n]} r\right]
$$

i.e.,

$$
\log ^{[p]} M(r, f \circ g) \leqslant\left(\rho^{(p, q)}(f)+\varepsilon\right) \log ^{[q-m+n]} r+O(1)
$$

and for a sequence of positive numbers of $r$ tending to infinity that

$$
\log ^{[p]}|f \circ g|(r) \leqslant\left(\rho^{(p, q)}(f)+\varepsilon\right) \log ^{[q-m+n]} r+O(1) .
$$

Also for the same reasoning, from (41) for a sequence of positive numbers of $r$ tending to infinity we obtain that

$$
\log ^{[p]}|f \circ g|(r) \leqslant\left(\lambda^{(p, q)}(f)+\varepsilon\right) \log ^{[q-m+n]} r+O(1) .
$$

Further from (42), for all sufficiently large positive numbers of $r$ it follows that

$$
\log ^{[p]}|f \circ g|(r) \geq\left(\lambda^{(p, q)}(f)-\varepsilon\right) \log ^{[q-m+n]} r+O(1),
$$

and for a sequence of positive numbers of $r$ tending to infinity that

$$
\log ^{[p]}|f \circ g|(r) \geq\left(\lambda^{(p, q)}(f)-\varepsilon\right) \log ^{[q-m+n]} r+O(1) .
$$

Moreover from (43) for a sequence of positive numbers of $r$ tending to infinity we obtain that

$$
\log ^{[p]}|f \circ g|(r) \geq\left(\rho^{(p, q)}(f)-\varepsilon\right) \log { }^{[q-m+n]} r+O(1) .
$$

Now from (37) and (70), for all sufficiently large positive numbers of $r$ we have that

$$
\frac{\log ^{[p]}|f \circ g|(r)}{\log ^{[p]}|f|\left(\exp ^{[m-n]} r\right)} \leqslant \frac{\left(\rho^{(p, q)}(f)+\varepsilon\right) \log ^{[q-m+n]} r+O(1)}{\left(\lambda^{(p, q)}(f)-\varepsilon\right) \log ^{[q-m+n]} r}
$$

i.e.,

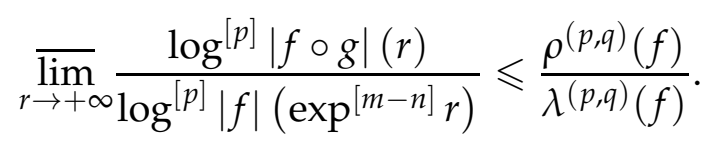


Similarly, from (38) and (70) for a sequence of positive numbers of $r$ tending to infinity it follows that

$$
\frac{\log ^{[p]}|f \circ g|(r)}{\log ^{[p]}|f|\left(\exp ^{[m-n]} r\right)} \leqslant \frac{\left(\rho^{(p, q)}(f)+\varepsilon\right) \log ^{[q-m+n]} r+O(1)}{\left(\rho^{(p, q)}(f)-\varepsilon\right) \log ^{[q-m+n]} r}
$$

i.e.,

$$
\varlimsup_{r \rightarrow+\infty} \frac{\log ^{[p]}|f \circ g|(r)}{\log ^{[p]}|f|\left(\exp ^{[m-n]} r\right)} \leqslant 1 .
$$

Also from (37) and (71) for a sequence of positive numbers of $r$ tending to infinity we obtain

$$
\frac{\log ^{[p]}|f \circ g|(r)}{\log ^{[p]}|f|\left(\exp ^{[m-n]} r\right)} \leqslant \frac{\left(\rho^{(p, q)}(f)+\varepsilon\right) \log ^{[q-m+n]} r+O(1)}{\left(\lambda^{(p, q)}(f)-\varepsilon\right) \log ^{[q-m+n]} r}
$$

i.e.,

$$
\varliminf_{r \rightarrow+\infty} \frac{\log ^{[p]} M(r, f \circ g)}{\log ^{[p]} M\left(\exp ^{[m-n]} r, f\right)} \leqslant \frac{\rho^{(p, q)}(f)}{\lambda^{(p, q)}(f)},
$$

and from (37) and (72) for a sequence of positive numbers of $r$ tending to infinity also we have

$$
\frac{\log ^{[p]}|f \circ g|(r)}{\log ^{[p]}|f|\left(\exp ^{[m-n]} r\right)} \leqslant \frac{\left(\lambda^{(p, q)}(f)+\varepsilon\right) \log ^{[q-m+n]} r+O(1)}{\left(\lambda^{(p, q)}(f)-\varepsilon\right) \log ^{[q-m+n]} r}
$$

i.e.,

$$
\varliminf_{r \rightarrow+\infty} \frac{\log ^{[p]} M(r, f \circ g)}{\log ^{[p]} M\left(\exp ^{[m-n]} r, f\right)} \leqslant 1 .
$$

Thus from (77), (78) and (79) it follows that

$$
\varliminf_{r \rightarrow+\infty} \frac{\log ^{[p]}|f \circ g|(r)}{\log ^{[p]}|f|\left(\exp ^{[m-n]} r\right)} \leqslant \min \left\{1, \frac{\rho^{(p, q)}(f)}{\lambda^{(p, q)}(f)}\right\} .
$$

Further from (36) and (73), for all sufficiently large positive numbers of $r$ we have that

$$
\frac{\log ^{[p]}|f \circ g|(r)}{\log ^{[p]}|f|\left(\exp ^{[m-n]} r\right)} \geq \frac{\left(\lambda^{(p, q)}(f)-\varepsilon\right) \log ^{[q-m+n]} r+O(1)}{\left(\rho^{(p, q)}(f)+\varepsilon\right) \log ^{[q-m+n]} r}
$$

i.e.,

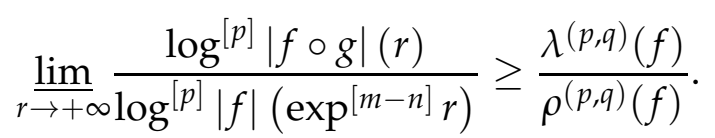

Similarly, from (39) and (73) for a sequence of positive numbers of $r$ tending to infinity it follows that

$$
\frac{\log ^{[p]}|f \circ g|(r)}{\log ^{[p]}|f|\left(\exp ^{[m-n]} r\right)} \geq \frac{\left(\lambda^{(p, q)}(f)-\varepsilon\right) \log ^{[q-m+n]} r+O(1)}{\left(\lambda^{(p, q)}(f)+\varepsilon\right) \log ^{[q-m+n]} r}
$$

i.e.,

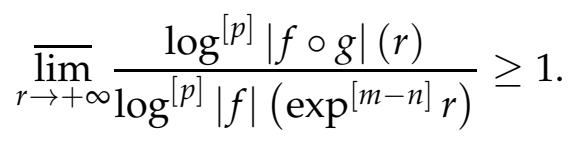


Also from (36) and (74), for a sequence of positive numbers of $r$ tending to infinity we obtain

$$
\frac{\log ^{[p]}|f \circ g|(r)}{\log ^{[p]}|f|\left(\exp ^{[m-n]} r\right)} \geq \frac{\left(\lambda^{(p, q)}(f)-\varepsilon\right) \log ^{[q-m+n]} r+O(1)}{\left(\rho^{(p, q)}(f)+\varepsilon\right) \log ^{[q-m+n]} r}
$$

i.e.,

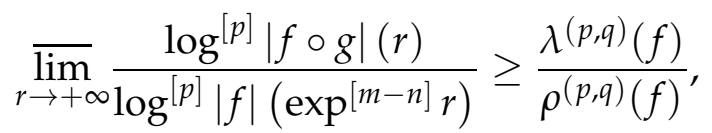

and from (36) and (75) for a sequence of positive numbers of $r$ tending to infinity also we have

$$
\frac{\log ^{[p]}|f \circ g|(r)}{\log ^{[p]}|f|\left(\exp ^{[m-n]} r\right)} \geq \frac{\left(\rho^{(p, q)}(f)-\varepsilon\right) \log ^{[q-m+n]} r+O(1)}{\left(\rho^{(p, q)}(f)+\varepsilon\right) \log ^{[q-m+n]} r}
$$

i.e.,

$$
\varlimsup_{r \rightarrow+\infty} \frac{\log ^{[p]}|f \circ g|(r)}{\log ^{[p]}|f|\left(\exp ^{[m-n]} r\right)} \geq 1 .
$$

Thus from (82), (83) and (84) it follows that

$$
\varlimsup_{r \rightarrow+\infty} \frac{\log ^{[p]}|f \circ g|(r)}{\log ^{[p]}|f|\left(\exp ^{[m-n]} r\right)} \geq \max \left\{1, \frac{\lambda^{(p, q)}(f)}{\rho^{(p, q)}(f)}\right\} .
$$

Hence the third part of the theorem follows from (76), (80), (65) and (85).

Case IV. Let $m>q=n$. Then from (40) for all sufficiently large positive numbers of $r$ we have

$$
\log ^{[p+m-q]}|f \circ g|(r) \leqslant\left(\rho^{(m, n)}(g)+\varepsilon\right) \log ^{[n]} r+O(1),
$$

and for a sequence of positive numbers of $r$ tending to infinity that

$$
\log ^{[p+m-q]}|f \circ g|(r) \leqslant\left(\lambda^{(m, n)}(g)+\varepsilon\right) \log ^{[n]} r+O(1) .
$$

Also from (41) for a sequence of positive numbers of $r$ tending to infinity we obtain that

$$
\log [p+m-q]|f \circ g|(r) \leqslant\left(\rho^{(m, n)}(g)+\varepsilon\right) \log ^{[n]} r+O(1) .
$$

Further, from (42) for all sufficiently large positive numbers of $r$ it follows that

$$
\log ^{[p+m-q]}|f \circ g|(r) \geq\left(\lambda^{(m, n)}(g)-\varepsilon\right) \log ^{[n]} r+O(1),
$$

and for a sequence of positive numbers of $r$ tending to infinity that

$$
\log [p+m-q]|f \circ g|(r) \geq\left(\rho^{(m, n)}(g)-\varepsilon\right) \log ^{[n]} r+O(1) .
$$

Moreover, from (43) for a sequence of positive numbers of $r$ tending to infinity we obtain that

$$
\log ^{[p+m-q]}|f \circ g|(r) \geq\left(\lambda^{(m, n)}(g)-\varepsilon\right) \log ^{[n]} r+O(1) .
$$


Therefore from (37) and (86), for all sufficiently large positive numbers of $r$ we have that

$$
\frac{\log ^{[p+m-q]}|f \circ g|(r)}{\log ^{[p]}|f|(r)} \leqslant \frac{\left(\rho^{(m, n)}(g)+\varepsilon\right) \log ^{[n]} r+O(1)}{\left(\lambda^{(p, q)}(f)-\varepsilon\right) \log ^{[q]} r}=\frac{\left(\rho^{(m, n)}(g)+\varepsilon\right) \log ^{[q]} r+O(1)}{\left(\lambda^{(p, q)}(f)-\varepsilon\right) \log ^{[q]} r}
$$

i.e.,

$$
\varlimsup_{r \rightarrow+\infty} \frac{\log ^{[p+m-q]}|f \circ g|(r)}{\log ^{[p]}|f|(r)} \leqslant \frac{\rho^{(m, n)}(g)}{\lambda^{(p, q)}(f)} .
$$

Similarly, from (38) and (86) for a sequence of positive numbers of $r$ tending to infinity it follows that

$$
\frac{\log ^{[p+m-q]}|f \circ g|(r)}{\log ^{[p]}|f|(r)} \leqslant \frac{\left(\rho^{(m, n)}(g)+\varepsilon\right) \log ^{[n]} r+O(1)}{\left(\rho^{(p, q)}(f)-\varepsilon\right) \log ^{[q]} r}=\frac{\left(\rho^{(m, n)}(g)+\varepsilon\right) \log ^{[q]} r+O(1)}{\left(\rho^{(p, q)}(f)-\varepsilon\right) \log ^{[q]} r}
$$

i.e.,

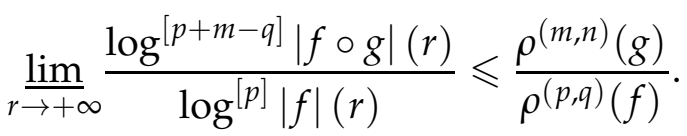

Also from (37) and (87) for a sequence of positive numbers of $r$ tending to infinity we obtain

$$
\frac{\log ^{[p+m-q]}|f \circ g|(r)}{\log ^{[p]}|f|(r)} \leqslant \frac{\left(\lambda^{(m, n)}(g)+\varepsilon\right) \log ^{[n]} r+O(1)}{\left(\lambda^{(p, q)}(f)-\varepsilon\right) \log ^{[q]} r}=\frac{\left(\lambda^{(m, n)}(g)+\varepsilon\right) \log ^{[q]} r+O(1)}{\left(\lambda^{(p, q)}(f)-\varepsilon\right) \log ^{[q]} r}
$$

i.e.,

$$
\varliminf_{r \rightarrow+\infty} \frac{\log ^{[p+m-q]}|f \circ g|(r)}{\log ^{[p]}|f|(r)} \leqslant \frac{\lambda^{(m, n)}(g)}{\lambda^{(p, q)}(f)}
$$

and from (37) and (88) for a sequence of positive numbers of $r$ tending to infinity also we have

$$
\frac{\log ^{[p+m-q]}|f \circ g|(r)}{\log ^{[p]}|f|(r)} \leqslant \frac{\left(\rho^{(m, n)}(g)+\varepsilon\right) \log ^{[n]} r+O(1)}{\left(\lambda^{(p, q)}(f)-\varepsilon\right) \log ^{[q]} r}=\frac{\left(\rho^{(m, n)}(g)+\varepsilon\right) \log ^{[q]} r+O(1)}{\left(\lambda^{(p, q)}(f)-\varepsilon\right) \log ^{[q]} r}
$$

i.e.,

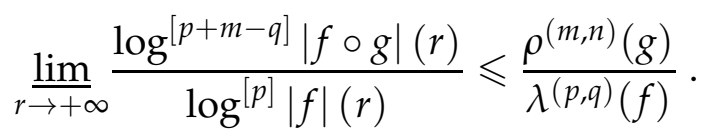

Thus from (93), (94) and (95) it follows that

$$
\varliminf_{r \rightarrow+\infty} \frac{\log ^{[p+m-q]}|f \circ g|(r)}{\log ^{[p]}|f|(r)} \leqslant \min \left\{\frac{\rho^{(m, n)}(g)}{\rho^{(p, q)}(f)}, \frac{\lambda^{(m, n)}(g)}{\lambda^{(p, q)}(f)}, \frac{\rho^{(m, n)}(g)}{\lambda^{(p, q)}(f)}\right\} .
$$

Further from (36) and (89), for all sufficiently large positive numbers of $r$ we have that

$$
\frac{\log ^{[p+m-q]}|f \circ g|(r)}{\log ^{[p]}|f|(r)} \geq \frac{\left(\lambda^{(m, n)}(g)-\varepsilon\right) \log ^{[n]} r+O(1)}{\left(\rho^{(p, q)}(f)+\varepsilon\right) \log ^{[q]} r}=\frac{\left(\lambda^{(m, n)}(g)-\varepsilon\right) \log ^{[q]} r+O(1)}{\left(\rho^{(p, q)}(f)+\varepsilon\right) \log ^{[q]} r}
$$

i.e.,

$$
\varliminf_{r \rightarrow+\infty} \frac{\log ^{[p+m-q]}|f \circ g|(r)}{\log ^{[p]}|f|(r)} \geq \frac{\lambda^{(m, n)}(g)}{\rho^{(p, q)}(f)} .
$$


Similarly, from (39) and (89) for a sequence of positive numbers of $r$ tending to infinity it follows that

$$
\frac{\log ^{[p+m-q]}|f \circ g|(r)}{\log ^{[p]}|f|(r)} \geq \frac{\left(\lambda^{(m, n)}(g)-\varepsilon\right) \log ^{[n]} r+O(1)}{\left(\lambda^{(p, q)}(f)+\varepsilon\right) \log ^{[q]} r}=\frac{\left(\lambda^{(m, n)}(g)-\varepsilon\right) \log ^{[q]} r+O(1)}{\left(\lambda^{(p, q)}(f)+\varepsilon\right) \log ^{[q]} r}
$$

i.e.,

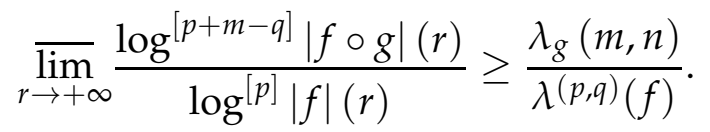

Also from(36) and (90) for a sequence of positive numbers of $r$ tending to infinity we obtain

$$
\frac{\log ^{[p+m-q]}|f \circ g|(r)}{\log ^{[p]}|f|(r)} \geq \frac{\left(\rho^{(m, n)}(g)-\varepsilon\right) \log ^{[n]} r+O(1)}{\left(\rho^{(p, q)}(f)+\varepsilon\right) \log ^{[q]} r}=\frac{\left(\rho^{(m, n)}(g)-\varepsilon\right) \log ^{[q]} r+O(1)}{\left(\rho^{(p, q)}(f)+\varepsilon\right) \log ^{[q]} r}
$$

i.e.,

$$
\varlimsup_{r \rightarrow+\infty} \frac{\log ^{[p+m-q]}|f \circ g|(r)}{\log ^{[p]}|f|(r)} \geq \frac{\rho^{(m, n)}(g)}{\rho^{(p, q)}(f)},
$$

and from (36) and (91) for a sequence of positive numbers of $r$ tending to infinity also we have

$$
\frac{\log ^{[p+m-q]}|f \circ g|(r)}{\log ^{[p]}|f|(r)} \geq \frac{\left(\lambda^{(m, n)}(g)-\varepsilon\right) \log ^{[n]} r+O(1)}{\left(\rho^{(p, q)}(f)+\varepsilon\right) \log ^{[q]} r}=\frac{\left(\lambda^{(m, n)}(g)-\varepsilon\right) \log ^{[q]} r+O(1)}{\left(\rho^{(p, q)}(f)+\varepsilon\right) \log ^{[q]} r}
$$

i.e.,

$$
\varlimsup_{r \rightarrow+\infty} \frac{\log ^{[p+m-q]}|f \circ g|(r)}{\log ^{[p]}|f|(r)} \geq \frac{\lambda^{(m, n)}(g)}{\rho^{(p, q)}(f)} .
$$

Thus from (98), (99) and (100) it follows that

$$
\varlimsup_{r \rightarrow+\infty} \frac{\log [p+m-q]}{\log ^{[p]}|f \circ g|(r)} \geq \max \left\{\frac{\lambda^{(m, n)}(g)}{\lambda^{(p, q)}(f)}, \frac{\rho^{(m, n)}(g)}{\rho^{(p, q)}(f)}, \frac{\lambda^{(m, n)}(g)}{\rho^{(p, q)}(f)}\right\} .
$$

Therefore the fourth part of the theorem follows from (92), (96), (98) and (101).

Case V. Let $m>q>n$. Currently from (37) and (86), we have for all sufficiently large positive numbers of $r$ that

$$
\frac{\log ^{[p+m-q]}|f \circ g|(r)}{\log ^{[p]}|f|\left(\exp ^{[q-n]} r\right)} \leqslant \frac{\left(\rho^{(m, n)}(g)+\varepsilon\right) \log ^{[n]} r+O(1)}{\left(\lambda^{(p, q)}(f)-\varepsilon\right) \log ^{[n]} r}
$$

i.e.,

$$
\varlimsup_{r \rightarrow+\infty} \frac{\log ^{[p+m-q]}|f \circ g|(r)}{\log ^{[p]}|f|\left(\exp ^{[q-n]} r\right)} \leqslant \frac{\rho^{(m, n)}(g)}{\lambda^{(p, q)}(f)} .
$$

Similarly, from (38) and (86) for a sequence of positive numbers of $r$ tending to infinity it follows that

$$
\frac{\log ^{[p+m-q]}|f \circ g|(r)}{\log ^{[p]}|f|\left(\exp ^{[q-n]} r\right)} \leqslant \frac{\left(\rho^{(m, n)}(g)+\varepsilon\right) \log ^{[n]} r+O(1)}{\left(\rho^{(p, q)}(f)-\varepsilon\right) \log ^{[n]} r}
$$


i.e.,

$$
\varliminf_{r \rightarrow+\infty} \frac{\log ^{[p+m-q]}|f \circ g|(r)}{\log ^{[p]}|f|\left(\exp ^{[q-n]} r\right)} \leqslant \frac{\rho^{(m, n)}(g)}{\rho^{(p, q)}(f)} .
$$

Also from (37) and (87), for a sequence of positive numbers of $r$ tending to infinity we obtain that

$$
\frac{\log ^{[p+m-q]}|f \circ g|(r)}{\log ^{[p]}|f|\left(\exp ^{[q-n]} r\right)} \leqslant \frac{\left(\lambda^{(m, n)}(g)+\varepsilon\right) \log ^{[n]} r+O(1)}{\left(\lambda^{(p, q)}(f)-\varepsilon\right) \log ^{[n]} r}
$$

i.e.,

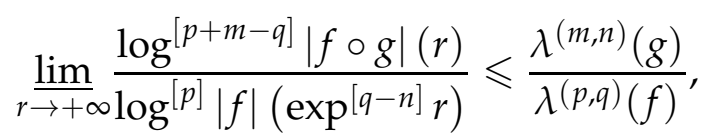

and from (37) and (88) for a sequence of positive numbers of $r$ tending to infinity also we have

$$
\frac{\log ^{[p+m-q]}|f \circ g|(r)}{\log ^{[p]}|f|\left(\exp ^{[q-n]} r\right)} \leqslant \frac{\left(\rho^{(m, n)}(g)+\varepsilon\right) \log ^{[n]} r+O(1)}{\left(\lambda^{(p, q)}(f)-\varepsilon\right) \log ^{[n]} r}
$$

i.e.,

$$
\varliminf_{r \rightarrow+\infty} \frac{\log ^{[p+m-q]}|f \circ g|(r)}{\log ^{[p]}|f|\left(\exp ^{[q-n]} r\right)} \leqslant \frac{\rho^{(m, n)}(g)}{\lambda^{(p, q)}(f)} .
$$

Thus from (103), (104) and (105) it follows that

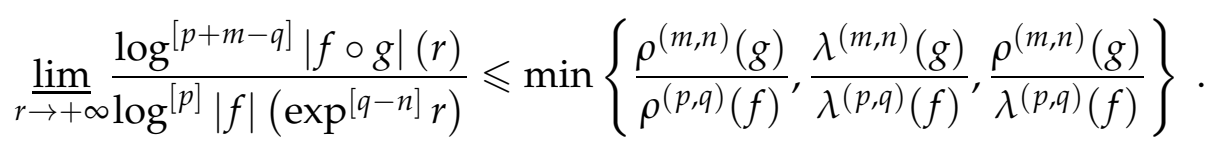

Further from (36) and (89), for all sufficiently large positive numbers of $r$ we have that

$$
\frac{\log ^{[p+m-q]}|f \circ g|(r)}{\log ^{[p]}|f|\left(\exp ^{[q-n]} r\right)} \geq \frac{\left(\lambda^{(m, n)}(g)-\varepsilon\right) \log ^{[n]} r+O(1)}{\left(\rho^{(p, q)}(f)+\varepsilon\right) \log ^{[n]} r}
$$

i.e.,

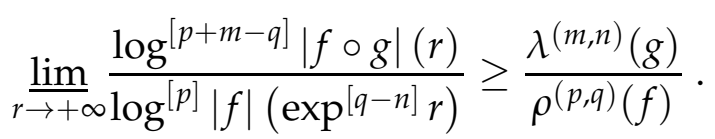

Similarly, from (39) and (89) for a sequence of positive numbers of $r$ tending to infinity it follows that

$$
\frac{\log ^{[p+m-q]}|f \circ g|(r)}{\log ^{[p]}|f|\left(\exp ^{[q-n]} r\right)} \geq \frac{\left(\lambda^{(m, n)}(g)-\varepsilon\right) \log ^{[n]} r+O(1)}{\left(\lambda^{(p, q)}(f)+\varepsilon\right) \log ^{[n]} r}
$$

i.e.,

$$
\varlimsup_{r \rightarrow+\infty} \frac{\log ^{[p+m-q]}|f \circ g|(r)}{\log ^{[p]}|f|\left(\exp ^{[q-n]} r\right)} \geq \frac{\lambda^{(m, n)}(g)}{\lambda^{(p, q)}(f)} .
$$

Also from (36) and (90), for a sequence of positive numbers of $r$ tending to infinity we obtain

$$
\frac{\log ^{[p+m-q]}|f \circ g|(r)}{\log ^{[p]}|f|\left(\exp ^{[q-n]} r\right)} \geq \frac{\left(\rho^{(m, n)}(g)-\varepsilon\right) \log ^{[n]} r+O(1)}{\left(\rho^{(p, q)}(f)+\varepsilon\right) \log ^{[n]} r}
$$


i.e.,

$$
\varlimsup_{r \rightarrow+\infty} \frac{\log ^{[p+m-q]}|f \circ g|(r)}{\log ^{[p]}|f|\left(\exp ^{[q-n]} r\right)} \geq \frac{\rho^{(m, n)}(g)}{\rho^{(p, q)}(f)},
$$

and from (36) and (91) for a sequence of positive numbers of $r$ tending to infinity also we have

$$
\frac{\log ^{[p+m-q]}|f \circ g|(r)}{\log ^{[p]}|f|\left(\exp ^{[q-n]} r\right)} \geq \frac{\left(\lambda^{(m, n)}(g)-\varepsilon\right) \log ^{[n]} r+O(1)}{\left(\rho^{(p, q)}(f)+\varepsilon\right) \log ^{[n]} r}
$$

i.e.,

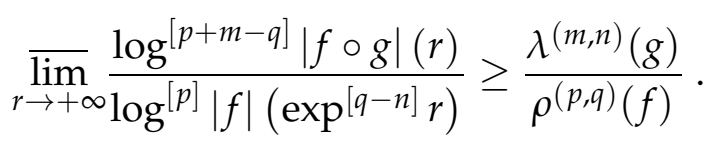

Thus from (98), (99), and (100) it follows that

$$
\varlimsup_{r \rightarrow+\infty} \frac{\log ^{[p+m-q]}|f \circ g|(r)}{\log ^{[p]}|f|\left(\exp ^{[q-n]} r\right)} \geq \max \left\{\frac{\lambda^{(m, n)}(g)}{\lambda^{(p, q)}(f)}, \frac{\rho^{(m, n)}(g)}{\rho^{(p, q)}(f)}, \frac{\lambda^{(m, n)}(g)}{\rho^{(p, q)}(f)}\right\} .
$$

Thus the fifth part of the theorem follows from (102), (106), (107) and (111).

Case VI. Let $m>q<n$. At this instant case from (37) and (86) for all sufficiently large positive numbers of $r$ we have that

$$
\frac{\log ^{[p+m-q]}|f \circ g|\left(\exp ^{[n-q]} r\right)}{\log ^{[p]}|f|(r)} \leqslant \frac{\left(\rho^{(m, n)}(g)+\varepsilon\right) \log [q] r+O(1)}{\left(\lambda^{(p, q)}(f)-\varepsilon\right) \log ^{[q]} r}
$$

i.e.,

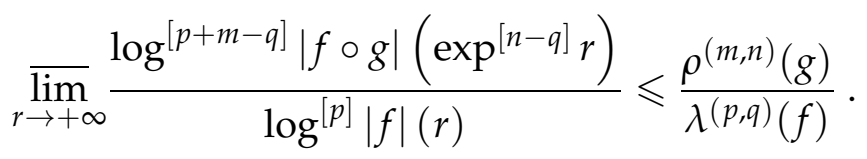

Similarly, from (38) and (86) for a sequence of positive numbers of $r$ tending to infinity it follows that

$$
\frac{\log ^{[p+m-q]}|f \circ g|\left(\exp ^{[n-q]} r\right)}{\log ^{[p]}|f|(r)} \leqslant \frac{\left(\rho^{(m, n)}(g)+\varepsilon\right) \log ^{[q]} r+O(1)}{\left(\rho^{(p, q)}(f)-\varepsilon\right) \log ^{[q]} r}
$$

i.e.,

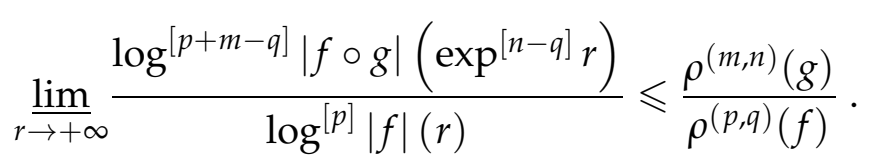

Also from (37) and (87) for a sequence of positive numbers of $r$ tending to infinity we obtain

$$
\frac{\log ^{[p+m-q]}|f \circ g|\left(\exp ^{[n-q]} r\right)}{\log ^{[p]}|f|(r)} \leqslant \frac{\left(\lambda^{(m, n)}(g)+\varepsilon\right) \log [q] r+O(1)}{\left(\lambda^{(p, q)}(f)-\varepsilon\right) \log ^{[q]} r}
$$

i.e.,

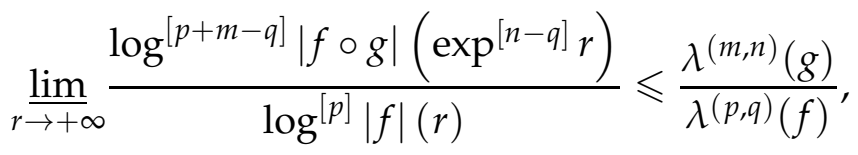


and from (37) and (88) for a sequence of positive numbers of $r$ tending to infinity also we have

$$
\frac{\log ^{[p+m-q]}|f \circ g|\left(\exp ^{[n-q]} r\right)}{\log ^{[p]}|f|(r)} \leqslant \frac{\left(\rho^{(m, n)}(g)+\varepsilon\right) \log ^{[q]} r+O(1)}{\left(\lambda^{(p, q)}(f)-\varepsilon\right) \log ^{[q]} r}
$$

i.e.,

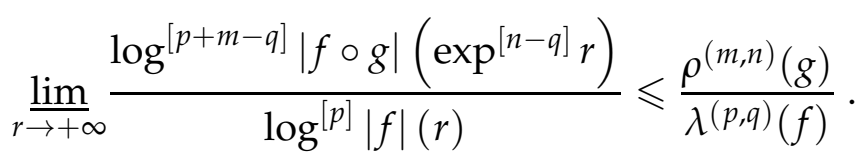

Thus from (113), (114) and (115) it follows that

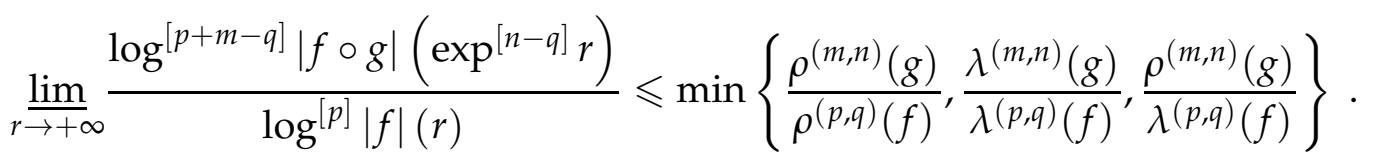

Further from (36) and (89), for all sufficiently large positive numbers of $r$ we have that

$$
\frac{\log ^{[p+m-q]}|f \circ g|\left(\exp ^{[n-q]} r\right)}{\log ^{[p]}|f|(r)} \geq \frac{\left(\lambda^{(m, n)}(g)-\varepsilon\right) \log ^{[q]} r+O(1)}{\left(\rho^{(p, q)}(f)+\varepsilon\right) \log ^{[q]} r}
$$

i.e.,

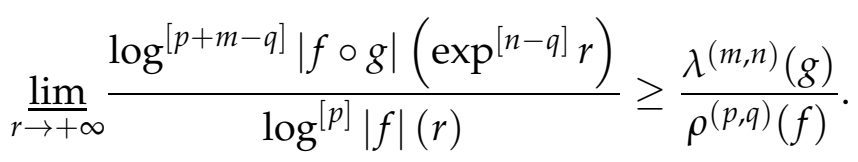

Similarly, from (39) and (89) for a sequence of positive numbers of $r$ tending to infinity it follows that

$$
\frac{\log ^{[p+m-q]}|f \circ g|\left(\exp ^{[n-q]} r\right)}{\log ^{[p]}|f|(r)} \geq \frac{\left(\lambda^{(m, n)}(g)-\varepsilon\right) \log ^{[q]} r+O(1)}{\left(\lambda^{(p, q)}(f)+\varepsilon\right) \log ^{[q]} r}
$$

i.e.,

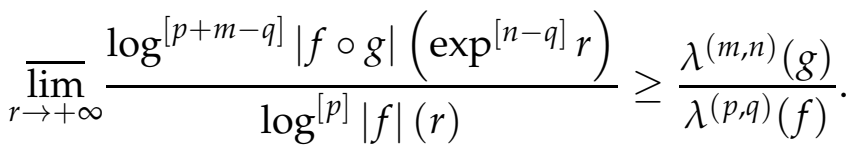

Also from (36) and (90), for a sequence of positive numbers of $r$ tending to infinity we obtain

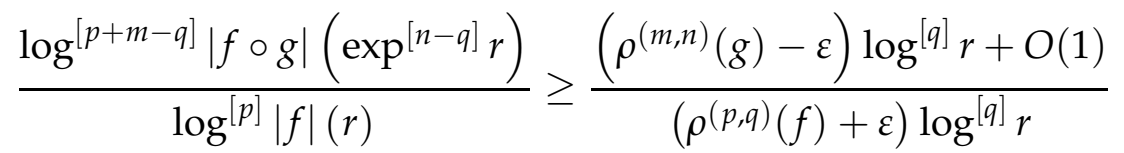

i.e.,

$$
\varlimsup_{r \rightarrow+\infty} \frac{\log ^{[p+m-q]}|f \circ g|\left(\exp ^{[n-q]} r\right)}{\log ^{[p]}|f|(r)} \geq \frac{\rho^{(m, n)}(g)}{\rho^{(p, q)}(f)}
$$

and from (36) and (91) for a sequence of positive numbers of $r$ tending to infinity also we have

$$
\frac{\log ^{[p+m-q]}|f \circ g|\left(\exp ^{[n-q]} r\right)}{\log ^{[p]}|f|(r)} \geq \frac{\left(\lambda^{(m, n)}(g)-\varepsilon\right) \log [q] r+O(1)}{\left(\rho^{(p, q)}(f)+\varepsilon\right) \log ^{[q]} r}
$$


i.e.,

$$
\varlimsup_{r \rightarrow+\infty} \frac{\log ^{[p+m-q]}|f \circ g|\left(\exp ^{[n-q]} r\right)}{\log ^{[p]}|f|(r)} \geq \frac{\lambda^{(m, n)}(g)}{\rho^{(p, q)}(f)} .
$$

Thus from (98), (99) and (100) it follows that

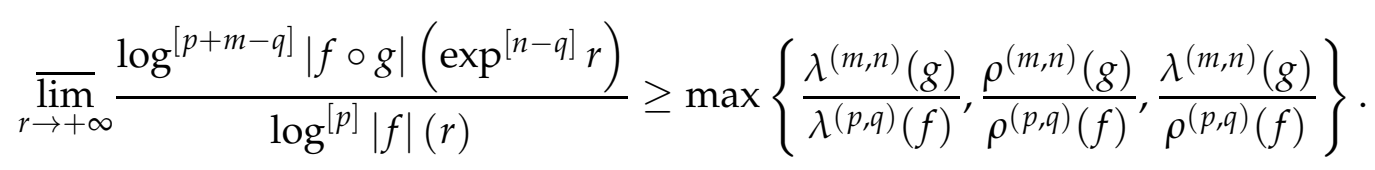

Hence the sixth part of the theorem follows from (112), (116), (118) and (121).

Theorem 8. Let $f, g \in \mathcal{A}(\mathbb{K})$ be such that $0<\lambda^{(p, q)}(f) \leq \rho^{(p, q)}(f)<\infty$ and $0<\lambda^{(m, n)}(g) \leq$ $\rho^{(m, n)}(g)<\infty$, where $p, q, m, n \in \mathbb{N}$. Then

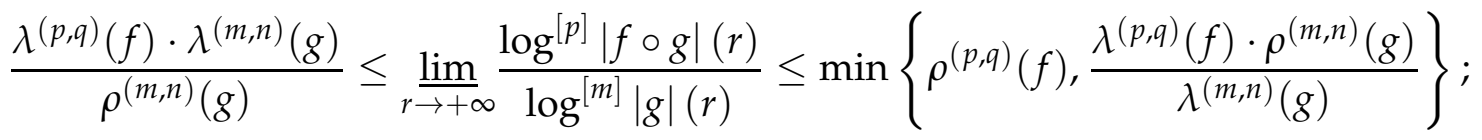

$$
\begin{aligned}
& \max \left\{\lambda^{(p, q)}(f), \frac{\rho^{(p, q)}(f) \cdot \lambda^{(m, n)}(g)}{\rho^{(m, n)}(g)}\right\} \leq \varlimsup_{r \rightarrow+\infty} \frac{\log [p]|f \circ g|(r)}{\log ^{[m]}|g|(r)} \leq \frac{\rho^{(p, q)}(f) \cdot \rho^{(m, n)}(g)}{\lambda^{(m, n)}(g)},
\end{aligned}
$$

when $q=m$,

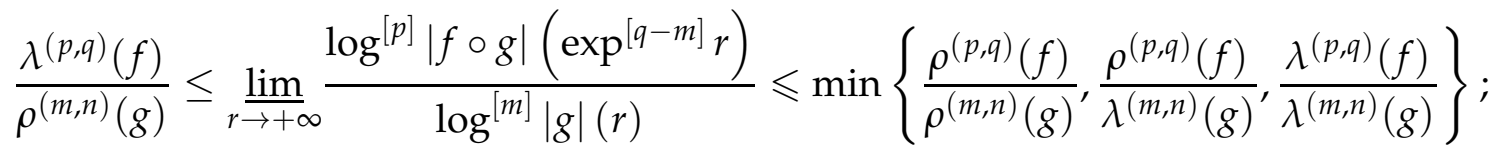

$$
\begin{aligned}
& \max \left\{\frac{\rho^{(p, q)}(f)}{\rho^{(m, n)}(g)}, \frac{\lambda^{(p, q)}(f)}{\rho^{(m, n)}(g)}, \frac{\lambda^{(p, q)}(f)}{\lambda^{(m, n)}(g)}\right\} \leq \varlimsup_{r \rightarrow+\infty} \frac{\log ^{[p]}|f \circ g|\left(\exp ^{[q-m]} r\right)}{\log ^{[m]}|g|(r)} \leq \frac{\rho^{(p, q)}(f)}{\lambda^{(m, n)}(g)},
\end{aligned}
$$

when $q>m$, and

$$
\frac{\lambda^{(m, n)}(g)}{\rho^{(m, n)}(g)} \leq \varliminf_{r \rightarrow+\infty} \frac{\log ^{[p+m-q]}|f \circ g|(r)}{\log ^{[m]}|g|(r)} \leq 1 \leq \varlimsup_{r \rightarrow+\infty} \frac{\log ^{[p+m-q]}|f \circ g|(r)}{\log ^{[m]}|g|(r)} \leq \frac{\rho^{(m, n)}(g)}{\lambda^{(m, n)}(g)^{2}},
$$

when $m>q$.

We omit the proof of Theorem 8 as it can easily be deduced in the line of Theorem 7 .

\section{REFERENCES}

[1] Bezivin J. P., Boussaf K., Escassut A. Zeros of the derivative of a p-adic meromorphic function. Bull. Sci. Math. 2012, 136 (8), 839-847.

[2] Bezivin J.P., Boussaf K., Escassut A. Some new and old results on zeros of the derivative of a p-adic meromorphic function. Contemp. Math. Amer. Math. Soc. 2013, 596, 23-30.

[3] Boussaf K., Escassut A., Ojeda J. Primitives of p-adic meromorphic functions. Contemp. Math. 2011, 551, 51-56.

[4] Boussaf K., Boutabaa A., Escassut A. Growth of p-adic entire functions and applications. Houston J. Math. 2014, 40 (3), 715-736.

[5] Boussaf K., Boutabaa A., Escassut A. Order, type and cotype of growth for p-adic entire functions: a survey with additional properties. p-Adic Numbers Ultrametric Anal. Appl. 2016, 8 (4), 280-297.

[6] Boutabaa A. Theorie de Nevanlinna p-adique. Manuscripta Math. 1990, 67, 251-269. 
[7] Chern T.Y.P. On the maximum modulus and the zeros of an transcendental entire function of finite logarithmic order. Bull. Hong Kong Math. Soc. 1999, 2, 271-278.

[8] Escassut A., Boussaf K., Boutabaa A. Order, type and cotype of growth for p-adic entire functions. Sarajevo J. Math. 2016, 12 (25) (2), suppl., 429-446.

[9] Escassut A. Analytic Elements in p-adic Analysis. World Scientic Publ. Pte. Ltd., Singapore, 1995.

[10] Escassut A. p-adic Value Distribution. Some Topics on Value Distribution and Differentability in Complex and P-adic Analysis. Math. Monogr., Beijing, 2008, 11, 42-138.

[11] Escassut A. Value Distribution in p-adic Analysis. World Scientic Publ. Pte. Ltd., Singapore, 2015.

[12] Escassut A., Ojeda J. Exceptional values of p-adic analytic functions and derivative. Complex Var. Elliptic Equ. 2011, 56 (1-4), 263-269.

[13] Escassut A., Ojeda J. Branched values and quasi-exceptional values for p-adic mermorphic functions. Houston J. Math. 2013, 39 (3), 781-795.

[14] Escassut A., Ojeda J. The p-adic Hayman conjecture when n=2. Complex Var. Elliptic Equ. 2014, 59 (10), $1452-$ 1455.

[15] Hu P.C., Yang C.C. Meromorphic Functions over non-Archimedean Fields. Kluwer Academic Publ., 2000.

[16] Juneja O. P., Kapoor G. P., Bajpai S. K. On the $(p, q)$-order and lower $(p, q)$-order of an entire function. J. Reine Angew. Math. 1976, 282, 53-67.

[17] Juneja O.P., Kapoor G.P., Bajpai S.K. On the $(p, q)$-type and lower $(p, q)$-type of an entire function. J. Reine Angew. Math. 1977, 290, 180-190.

[18] Robert A. A Course in p-Adic Analysis, Graduate texts. Springer, 2000.

[19] Ojeda J. On Hayman's Conjecture over a p-adic field, Taiwanese J. Math. 2008, 12 (9), 2295-2313.

Received 24.05.2018

Бісвас Т. Оцінка орієнтованого росту складених р-адичних цілих функцій, цио залежить від $(p, q)$-го порядку // Карпатські матем. публ. - 2018. - Т.10, №2. - С. 248-272.

Нехай $\mathbb{K}-$ повне ультраметричне алгебраїчно замкнуте поле, $\mathcal{A}(\mathbb{K})-\mathbb{K}$-алгебра цілих функцій на $\mathbb{K}$. Аля довільної $p$-адичної цілої функції $f \in \mathcal{A}(\mathbb{K})$ і $r>0$ позначимо $|f|(r)$ число $\sup \{|f(x)|:|x|=r\}$, де $|\cdot|(r) \in$ мультиплікативною нормою на $\mathcal{A}(\mathbb{K})$. $\Delta$ ля довільних двох цілих функцій $f \in \mathcal{A}(\mathbb{K})$ та $g \in \mathcal{A}(\mathbb{K})$ співвідношення $\frac{|f|(r)}{|g|(r)}$ при $r \rightarrow \infty$ називають порівняльним ростом $f$ відносно $g$ в сенсі їхніх мультиплікативних норм. Аналогічно до того, як це роблять в комплексному аналізі, в цій статті ми визначаємо поняття $(p, q)$-го порядку (відповідно $(p, q)$-го нижнього порядку) росту наступним чином $\rho^{(p, q)}(f)=\limsup _{r \rightarrow+\infty} \frac{\log ^{[p]}|f|(r)}{\log ^{[q]} r}$ (відпоідно $\left.\lambda^{(p, q)}(f)=\liminf _{r \rightarrow+\infty} \frac{\log [p]|f|(r)}{\log ^{[q]} r}\right)$, де $p$ і $q$ два довільні натуральні числа. Ми досліджуємо деякі властивості росту складених $p$-адичних цілих функцій на основі їхнього $(p, q)$-го порядку і $(p, q)$-го нижнього порядку.

Ключові слова і фрази: р-адична ціла функція, ріст, $(p, q)$-й порядок, $(p, q)$-й нижній порядок, композиція. 